\title{
HP-FINITE ELEMENT APPROXIMATIONS ON NON-MATCHING GRIDS FOR PARTIAL DIFFERENTIAL EQUATIONS WITH NON-NEGATIVE CHARACTERISTIC FORM
}

\begin{abstract}
Andrea Toselli ${ }^{1, *}$
Abstract. We propose and analyze a domain decomposition method on non-matching grids for partial differential equations with non-negative characteristic form. No weak or strong continuity of the finite element functions, their normal derivatives, or linear combinations of the two is imposed across the boundaries of the subdomains. Instead, we employ suitable bilinear forms defined on the common interfaces, typical of discontinuous Galerkin approximations. We prove an error bound which is optimal with respect to the mesh-size and suboptimal with respect to the polynomial degree. Our analysis is valid for arbitrary shape-regular meshes and arbitrary partitions into subdomains. Our method can be applied to advective, diffusive, and mixed-type equations, as well, and is well-suited for problems coupling hyperbolic and elliptic equations. We present some two-dimensional numerical results that support our analysis for the case of linear finite elements.
\end{abstract}

Mathematics Subject Classification. 65N12, 65N30, 65N35, 65N55.

Received: January 14, 2002.

\section{INTRODUCTION}

We consider the partial differential equation

$$
L u:=-\nabla \cdot(A \nabla u)+\mathbf{b} \cdot \nabla u+c u=f, \quad \text { in } \Omega,
$$

where $\Omega \subset \mathbb{R}^{n}, n=2,3$, is a bounded, connected, Lipschitz polygon or polyhedron, with outward unit normal $\mathbf{n}$. Here, $A$ is a symmetric, positive-semidefinite matrix in $\Omega, \mathbf{b}$ a given velocity field, $c$ a non-negative reaction coefficient that may arise from a finite difference discretization of a time derivative, and $f$ is a source term. In the next section, we make further hypotheses on $L$ and we introduce appropriate boundary conditions.

The aim of this paper is to construct and analyze an $h p$-finite element method for problem (1) on nonmatching grids. We propose an approach which is typical of discontinuous Galerkin (DG) methods, where finite element spaces consisting of discontinuous functions are considered. In particular, in a DG approach no

Keywords and phrases. Advection-diffusion, hyperbolic problems, stabilization, domain decomposition, non-matching grids, discontinuous Galerkin, $h p$-finite elements.

${ }^{1}$ Seminar for Applied Mathematics, ETH Zürich, Rämistrasse 101, 8092 Zürich, Switzerland. e-mail: toselli@sam.math.ethz.ch

* This work was carried out and completed while the author was affiliated to the Courant Institute of Mathematical Sciences, New York, and supported in part by the Applied Mathematical Sciences Program of the U.S. Department of Energy under Contract DEFGO288ER25053. 
continuity is imposed across the interelement boundaries and consistency is achieved by introducing suitable interface terms. The meshes need not be conforming, even if they cannot be completely arbitrary. A large variety of DG methods have been proposed and studied in the last thirty years. We refer to $[15]$, and to $[6,16]$ and to the references therein.

Even though our method is also valid for the pure diffusive case, our focus is on transport and transportdominated equations, and on problems where equations of different type are coupled.

Here, we mainly use the ideas of four previous works.

In $[6,25]$, a domain decomposition (DD) method for non-matching grids is developed for the Poisson equation using interface terms borrowed from a DG method. The main idea of this work is that DG methods can provide powerful approximations on non-matching grids, without the need to impose any kind of continuity across the subdomain boundaries. To our knowledge, these are the first works where these ideas are employed in a DD framework for non-matching grids. Our method uses the same idea as in [6,25], but we are interested in the more general problem (1). For this reason, we also consider the work in [16], where a DG $h p$-finite element method is proposed and studied for problem (1). Much of our analysis is similar to that in [16], but our focus is on DD on non-matching grids: given a general partition of $\Omega$ into subdomains, we consider local conforming, shaperegular meshes on them; such triangulations are completely independent from one another and, consequently, some of the interface contributions introduced in [16] need to be suitably modified in order to treat arbitrarily small intersections between element boundaries.

In this paper, we consider local conforming finite element spaces for problem (1). We have chosen the Streamline Diffusion (SD) studied in [17], which is a generalization of the original SD method in [20]. However, any other approximation method for problem (1) can be employed as an approximation of the local problems. In particular, we could use the same DG method as in [16] for some of the local problems.

We remark that even though we use a similar idea as in $[6,25]$, our method is different from theirs, when applied to a symmetric elliptic problem. In this case, we use a different interface contribution and do not obtain a symmetric bilinear form.

Our DD method presents the following characteristics:

1. it is valid for two- and three-dimensional problems;

2. it is valid for arbitrary partitions into non-overlapping subdomains (geometrically conforming and nonconforming, with arbitrarily small subdomains);

3. the local meshes are only required to be shape-regular and different polynomial spaces can be employed on the subdomains;

4. the error bound that we prove is $h$-optimal and $p$-suboptimal by half a power of $p$;

5. our method is valid for transport, diffusive, and mixed-type equations, and no a priori knowledge of the character of the equation solved is necessary;

6. it is also suitable for heterogeneous DD problems, where equations of different kinds are coupled (hyperbolic and elliptic, for instance), and does not require any a priori knowledge of the character of the local problems.

We remark that some of these characteristics are inherited from the original methods in $[16,17]$.

In the last ten years, many domain decomposition methods have been proposed for the approximation of second-order elliptic problems on non-matching grids. Among them, the mortar method has become more and more popular: see, e.g., $[7,8,10,11,22,27]$, for positive-definite scalar problems, and the references in [9], for a large number of other applications. As is standard practice, by a mortar method, we mean an approximation scheme where the jumps of a trace of the solution across the subdomain boundaries are required to be perpendicular to a suitable FE space (mortar space) defined on the interface, also including methods that are somewhat different from the original one in [11]. In particular, a considerable amount of work has been done for mixed approximations of positive-definite problems (see $[3,28]$ ). We also mention some other methods of non-mortar type for symmetric positive-definite problems: see, e.g., [13,14], and, as already mentioned, [6,25].

We believe that the case of approximations on non-matching grids of first-order transport equations is considerably simpler. Indeed, a close look at the original DG method for hyperbolic problems [21] and at some 
following generalizations [12] reveals that if the mesh is not conforming and the intersections between element boundaries are arbitrarily small the stability and error estimates remain valid. A simple DG approach based on classical upwinding appears to be the right choice for approximating the transport part of the equation on non-matching grids. This is the main idea underlying the method that we present here in the pure transport case.

The case of transport-dominated transport-diffusion problems or of equations coupling pure transport and diffusive regimes appears to be much harder and less well understood for approximations on non-matching grids. In [1], a DG approach for the transport part of the equation and a standard mortar approach for the diffusive part are combined. However, a mortar condition across the interfaces seems incompatible with the case of a vanishing or an almost vanishing viscosity. A proposed remedy for the diffusive part is to match two suitable families of fluxes (i.e., linear combinations of a function and its normal derivative) across the interfaces. This choice allows to treat the interfaces in a more symmetric way, preventing the non-physical situation where the value of the solution on the outflow boundary of a subdomain is determined by that on adjacent subdomains. In addition, a natural iteration by subdomain procedure can be devised for the solution of the resulting linear system, where local problems with Robin boundary conditions are solved. This approach has been explored in [4] and later in [2]. In [4], a finite element approximation is studied for a mixed formulation of problem of (1); such a method is not applicable to our problem since it requires that the convective term in (1) be small compared to the diffusive and reaction terms. In [2], a low-order finite volume approximation is considered for (1) where this restriction is removed, but the flux conditions imposed at the interfaces do not seem to be adequate for the pure transport case $(A=0)$, where simple upwinding is enough. In addition, there are some restrictions on the local meshes for the case of non-conforming partitions into subdomains. We finally mention [26, 29], where flux-matching conditions are considered for a class of non-linear degenerate parabolic problems arising in flows in porous media.

We believe that the method that we propose presents some considerable advantages compared to the abovementioned works.

The rest of the paper is organized as follows. In the next section, we introduce some hypotheses on the coefficients of the continuous problem (1) and some appropriate boundary conditions, and, in Section 3, we describe our $h p$-finite element approximation. Stability and error estimates are proved in 4 and 5 , respectively, under some restrictions on the diffusive matrix $A$. The case of a general matrix $A$ is treated in Section 6 . Finally, we present some numerical results for the case of linear finite elements in Section 7.

\section{Continuous problem}

We make the following hypotheses on the operator $L$ in $(1)$ :

$$
\begin{aligned}
& A=\left\{A_{i j}\right\} \in L^{\infty}(\Omega)^{n \times n}, \\
& \mathbf{b} \in W^{1, \infty}(\Omega)^{n} \\
& c \in L^{\infty}(\Omega)
\end{aligned}
$$

We also assume

$$
\begin{aligned}
& A_{i j}=A_{j i} ; \quad \mathbf{y}^{t} A \mathbf{y} \geq 0, \quad \mathbf{y} \in \mathbb{R}^{n}, \quad \text { a.e. in } \Omega \\
& \gamma:=c-\frac{1}{2} \nabla \cdot \mathbf{b} \geq \gamma_{0}>0, \quad \text { a.e. in } \Omega .
\end{aligned}
$$


We next introduce some boundary conditions and, following [16], we first partition the boundary $\partial \Omega$ as

$$
\begin{aligned}
& \partial \Omega_{0}:=\left\{x \in \partial \Omega \mid \mathbf{n}^{t} A \mathbf{n}>0\right\} \\
& \partial_{-} \Omega:=\left\{x \in \partial \Omega \backslash \partial \Omega_{0} \mid \quad \mathbf{b} \cdot \mathbf{n}<0\right\}, \\
& \partial_{+} \Omega:=\left\{x \in \partial \Omega \backslash \partial \Omega_{0} \mid \quad \mathbf{b} \cdot \mathbf{n} \geq 0\right\},
\end{aligned}
$$

and, further, $\partial \Omega_{0}$ as

$$
\partial \Omega_{0}=\partial \Omega_{D} \cup \partial \Omega_{N}
$$

We suppose that

$$
\mathbf{b} \cdot \mathbf{n} \geq 0, \quad \text { on } \partial \Omega_{N} .
$$

We then impose Dirichlet boundary conditions on $\partial \Omega_{D}$ and on the inflow boundary $\partial_{-} \Omega$, Neumann conditions on $\partial \Omega_{N}$, and no conditions on the outflow boundary $\partial_{+} \Omega$ :

$$
\begin{aligned}
& u=g_{D}, \quad \text { on } \partial \Omega_{D} \cup \partial_{-} \Omega \\
& \mathbf{n}^{t} A \nabla u=g_{N}, \quad \text { on } \partial \Omega_{N} .
\end{aligned}
$$

We note that additional hypotheses are needed on $A$ in order to define our boundary conditions. Since $A$ needs to be defined on the boundary, as in [16], we assume that $A$ is piecewise continuous on $\bar{\Omega}$.

We refer to [17], for a discussion of the well-posedness of problem (1) with the boundary conditions (8).

For any $\mathcal{D} \subset \Omega$, we define the bilinear form associated to the operator $L$ :

$$
a_{\mathcal{D}}(u, v):=\int_{\mathcal{D}}(A \nabla u \cdot \nabla v+\mathbf{b} \cdot \nabla u v+c u v) \mathrm{d} x, \quad u, v \in H^{1}(\Omega) .
$$

In the following, we only consider the case of Neumann and homogeneous Dirichlet conditions in full detail. The generalization of our method and its analysis to more general conditions is straightforward. We initially assume that the matrix $A$ is constant on each element of our triangulations (see the following two sections), and next discuss the modifications of our algorithms required for the case of a general $A$. We remark that in our numerical tests such modifications do not appear to be necessary.

\section{Approximations ON NON-MATCHING GRIDS}

We now introduce a non-conforming approximation of problem (1) with the boundary conditions (8). We first consider a non-overlapping partition of the domain $\Omega$

$$
\mathcal{F}_{H}=\left\{\Omega_{i} \mid \quad 1 \leq i \leq N, \quad \bigcup_{i=1}^{N} \overline{\Omega_{i}}=\bar{\Omega}\right\}
$$

such that each $\Omega_{i}$ is an open, connected, Lipschitz polygon or polyhedron. We denote the outward normal of $\Omega_{i}$ by $\mathbf{n}_{i}$. The elements of $\mathcal{F}_{H}$ are also called substructures. We stress the fact that we do not make any additional hypothesis on the partition $\mathcal{F}_{H}$.

We define the edges (or faces, if $n=3$ ) of the partition as the intersections $E_{i j}$, such that

$$
\bar{E}_{i j}:=\partial \Omega_{i} \cap \partial \Omega_{j}, \quad i \neq j, \quad m_{n-1}\left(E_{i j}\right)>0,
$$


where $m_{n-1}\left(E_{i j}\right)$ denotes the $(n-1)$-dimensional measure of $E_{i j}$ and $\bar{E}_{i j}$ its closure. Let $\mathcal{E}_{H}$ be the set of edges of $\mathcal{F}_{H}$, and let the interface $\Gamma$ be the union of the edges of $\mathcal{F}_{H}$, or, equivalently the parts of the subdomain boundaries that do not belong to $\partial \Omega$ :

$$
\Gamma:=\bigcup_{i=1}^{N} \partial \Omega_{i} \backslash \partial \Omega .
$$

For every subdomain $\Omega_{i}$, let $\mathcal{I}_{i}$ be the set consisting of $i$ and the indices $j$, such that $E_{i j}$ is an edge of $\Omega_{i}$ :

$$
\mathcal{I}_{i}:=\{i\} \cup\left\{j \mid E_{i j} \subset \partial \Omega_{i}, E_{i j} \in \mathcal{E}_{H}\right\}
$$

We next consider a triangulation $\mathcal{T}_{i}$ of each substructure $\Omega_{i}$, consisting of triangles or tetrahedra. Let $h_{t}$ be the diameter of the element $t \in \mathcal{T}_{i}$ and $h_{i}$ be the maximum of the diameters of the elements of $\mathcal{T}_{i}$. We assume that each $\mathcal{T}_{i}$ is shape-regular (see [23], Chap. 3). The triangulations considered do not need to match across the boundaries of the substructures and do not need to be quasi-uniform. Triangulations made of quadrilaterals or parallelepipeds are also possible.

For each $\Omega_{i}$, we then fix a polynomial degree $p_{i} \geq 1$ and introduce the local conforming space

$$
V_{i}:=\left\{u \in C^{0}\left(\Omega_{i}\right) \mid \quad u_{\left.\right|_{t}} \in \mathbb{P}_{p_{i}}(t), t \in \mathcal{T}_{i} ; \quad u=0 \quad \text { on } \partial_{-} \Omega \cup \partial \Omega_{D}\right\}
$$

where $\mathbb{P}_{p_{i}}(t)$ is the space of polynomials of maximum degree $p_{i}$ on $t$.

We make no continuity assumption for our global space:

$$
V:=\left\{u \in L^{2}(\Omega) \mid \quad u_{\left.\right|_{\Omega_{i}}} \in V_{i}, 1 \leq 1 \leq N ; \quad u=0 \quad \text { on } \partial_{-} \Omega \cup \partial \Omega_{D}\right\} .
$$

Given a function $u \in V$, we define the restriction

$$
u_{i}:=u_{\left.\right|_{\Omega_{i}}} \in V_{i}
$$

We first need to introduce some notations for an element $t$ and a substructure $\Omega_{i}$ :

$$
\begin{aligned}
\bar{b}_{t}:=\sup _{x \in t}|\mathbf{b}(x)|, \quad \bar{b}_{i}:=\sup _{x \in \Omega_{i}}|\mathbf{b}(x)|, \\
\bar{c}_{t}:=\|c\|_{t ; \infty}, \quad \bar{c}_{i}:=\|c\|_{\Omega_{i} ; \infty}, \\
\bar{\gamma}_{t}:=\|\gamma\|_{t ; \infty}, \quad \bar{\gamma}_{i}:=\|\gamma\|_{\Omega_{i} ; \infty},
\end{aligned}
$$

where $\gamma$ is defined in (6). In addition, we set

$$
\bar{a}_{t}:=\|\Lambda\|_{t ; \infty}, \quad \bar{a}_{i}:=\|\Lambda\|_{\Omega_{i} ; \infty},
$$

where $\Lambda(x)$ is the largest eigenvalue of the matrix $A(x)$ at the point $x$, and, finally,

$$
p_{t}:=p_{i}, \quad \text { if } t \subset \Omega_{i} .
$$

Our non-conforming approximation in $V$ is given in terms of the local stabilized bilinear forms for the StreamlineDiffusion approximation of $L$ and three additional bilinear forms that act on the traces of functions in $V$ on the interface $\Gamma$. The first is related to the principal part of the operator $L$, the second is borrowed from classical upwind schemes, and the third penalizes the jumps of the traces of a function across $\Gamma$. We stress the fact that we do not impose the (strong or weak) continuity of a function, its normal derivative, or a linear combination of the two, across the boundaries of the substructures, as is usually done in some of the mortar methods or some other approximations on non-matching grids (see Sect. 1). 
We now introduce some local bilinear forms. It is well known that for second order advection-dominated problems the original bilinear form $a(\cdot, \cdot)$ has to be modified in order to remove spurious oscillations of the Galerkin approximation on standard continuous polynomial spaces, if the mesh does not resolve boundary or internal layers. A large number of strategies have been proposed in the past twenty years and many of them consist of adding mesh dependent terms to the FE approximation; see, e.g., [19,23] and the references therein. Here, we consider the SD, or Streamline Upwind/Petrov-Galerkin, method developed in [17], but note that other methods can also be employed. We introduce the modified local bilinear forms

$$
a_{i}^{h}(u, v):=a_{\Omega_{i}}(u, v)+\sum_{t \subset \Omega_{i}} \delta(t) \int_{t} \hat{L} u(\mathbf{b} \cdot \nabla v) \mathrm{d} x, \quad u, v \in V_{i}, \quad i=1, \ldots, N
$$

where $\hat{L}:=L$ and, for each element $t, \delta(t)$ is a positive number.

We also need to introduce stabilized local right-hand sides. For a substructure $\Omega_{i}$, we define

$$
l_{i}^{h}(v):=\int_{\Omega_{i}} f v \mathrm{~d} x+\sum_{t \subset \Omega_{i}} \delta(t) \int_{t} f(\mathbf{b} \cdot \nabla v) \mathrm{d} x .
$$

In the following, we also use the same notation $\delta(x)$, for the corresponding function, defined a.e. on $\Omega$, that is equal to $\delta(t)$ if $x \in t$.

We next introduce three bilinear forms defined on $\Gamma$. The first is denoted by $s_{a}(\cdot, \cdot)$ and is related to the principal part of the operator $L$. We first extend the normal unit vector $\mathbf{n}$ to the interface $\Gamma$. Given an arbitrary but fixed ordering of the subdomains in $\mathcal{F}_{H}$, for $E_{i j} \in \mathcal{E}_{H}$, we set

$$
\mathbf{n}(x)_{\left.\right|_{E_{i j}}}:= \begin{cases}\mathbf{n}_{i}(x), & \text { if } i>j \\ \mathbf{n}_{j}(x), & \text { if } i<j\end{cases}
$$

We also define the jump $[v]$ of $v$ on the interface $\Gamma$. For $E_{i j} \in \mathcal{E}_{H}$, we set

$$
[v]_{\left.\right|_{E_{i j}}}:= \begin{cases}v_{i}-v_{j}, & \text { if } i>j \\ v_{j}-v_{i}, & \text { if } i<j\end{cases}
$$

where $v_{i}$ and $v_{j}$ are the values of $v$ on $\partial \Omega_{i}$ and $\partial \Omega_{j}$, respectively. We note that our definitions of normal and jumps depend on the particular ordering of $\mathcal{F}_{H}$ chosen.

We are now ready to define the bilinear form $s_{a}(\cdot, \cdot)$ :

For $u, v \in V$, we set

$$
s_{a}(u, v):=\sum_{E \in \mathcal{E}_{H}} \int_{E}([u]\langle A \nabla v \cdot \mathbf{n}\rangle-\langle A \nabla u \cdot \mathbf{n}\rangle[v]) \mathrm{d} s
$$

where

$$
\langle A \nabla v \cdot \mathbf{n}\rangle_{\left.\right|_{E_{i j}}}:=\frac{1}{2}\left(A_{i} \nabla v_{i} \cdot \mathbf{n}+A_{j} \nabla v_{j} \cdot \mathbf{n}\right), \quad E_{i j} \in \mathcal{E}_{H}
$$

with $A_{i}$ and $A_{j}$ the restrictions of $A$ to $\Omega_{i}$ and $\Omega_{j}$, respectively. We note that the definition of $s_{a}(\cdot, \cdot)$ is independent of the ordering of $\mathcal{F}_{H}$.

In order to define our second bilinear form $s_{b}(\cdot, \cdot)$ on $\Gamma$, we need to introduce some additional partitions of the subdomain boundaries. For a substructure $\Omega_{i}$, we define the two sets

$$
\begin{aligned}
& \partial_{-} \Omega_{i}:=\left\{x \in \partial \Omega_{i} \mid \quad \mathbf{b}(x) \cdot \mathbf{n}_{i}(x)<0\right\} \\
& \partial_{+} \Omega_{i}:=\left\{x \in \partial \Omega_{i} \mid\right. \\
& \left.\mathbf{b}(x) \cdot \mathbf{n}_{i}(x) \geq 0\right\}
\end{aligned}
$$


We note that these two sets do not form a partition of $\partial \Omega_{i} \backslash \partial \Omega$ in general, since the normal $\mathbf{n}_{i}$ is only defined almost everywhere, and, possibly, a set of zero $(n-1)$-dimensional measure is excluded. In addition, for almost every $x \in \partial_{-} \Omega_{i} \cap \Gamma$, there exists an index $j$, such that $x \in \partial_{+} \Omega_{j}$; we set

$$
u_{-i}(x):=u_{j}(x)
$$

We define the oriented jump on $\partial_{-} \Omega_{i} \cap \Gamma$ as

$$
\lfloor u\rfloor:=u_{i}-u_{-i}
$$

We note that $\lfloor u\rfloor$ and $[u]$ have the same absolute value, but may have opposite sign.

For $u, v \in V$, we set

$$
s_{b}(u, v):=\sum_{i=1}^{N} \int_{\partial-\Omega_{i} \cap \Gamma}\left|\mathbf{b} \cdot \mathbf{n}_{i}\right|\lfloor u\rfloor v_{i} \mathrm{~d} s .
$$

Our third bilinear form $s_{r}(\cdot, \cdot)$ penalizes the jumps of the traces of a function across $\Gamma$. It is defined as

$$
s_{r}(u, v):=\sum_{E \in \mathcal{E}_{H}} \int_{E} \sigma[u][v] \mathrm{d} s=\int_{\Gamma} \sigma[u][v] \mathrm{d} s .
$$

The choice of $\sigma$ is crucial. Here, we can generalize the definitions for the methods in [16] and [6]. The function $\sigma$ is a piecewise constant function on each edge $E_{i j} \in \mathcal{E}_{H}$. Given a segment $e \subset E_{i j}$, such that

$$
\bar{e}=\partial t \cap \partial t^{\prime}, \quad t \in \mathcal{T}_{i}, \quad t^{\prime} \in \mathcal{T}_{j}, \quad m_{n-1}(e)>0
$$

we set

$$
\sigma_{l_{e}}:=\frac{1}{2} \sigma_{0}\left(\frac{\bar{a}_{t} p_{t}}{h_{t}}+\frac{\bar{a}_{t^{\prime}} p_{t^{\prime}}}{h_{t^{\prime}}}\right)
$$

with $\sigma_{0}$ an arbitrary positive constant, which, for the purpose of the analysis, we assume to be one. Our definition generalizes that in [16] to the case where the intersection $e$ has arbitrarily small length; with our definition, $\sigma$ remains bounded. It also generalizes that in [6] to the case where two adjacent subdomains have very different meshes. We finally note that, as for the DG method in [16], the bilinear form $s_{r}(\cdot, \cdot)$ is identically zero in the pure hyperbolic case.

We are now ready to define our bilinear form and right-hand side on $V$. We set

$$
\begin{aligned}
a^{h}(u, v) & :=\sum_{i=1}^{N} a_{i}^{h}(u, v)+s_{a}(u, v)+s_{b}(u, v)+s_{r}(u, v), \quad u, v \in V, \\
l^{h}(v) & :=\sum_{i=1}^{N} l_{i}^{h}(v)+\int_{\partial \Omega_{N}} g_{N} v \mathrm{~d} s, \quad v \in V .
\end{aligned}
$$

Our discrete problem becomes: Find $u \in V$, such that

$$
a^{h}(u, v)=l^{h}(v), \quad v \in V
$$


Remark 3.1. We note that the bilinear form $a^{h}(\cdot, \cdot)$ consists of two main contributions: one consisting of local terms coming from the FE approximation of the operator $L$ on the subdomains, and one consisting of terms on the interface $\Gamma$ which ensure the consistency of this non-conforming approximation. For the first contribution and for the modified right-hand side, we have employed the SD method, but any other method for the approximation of $L$ could be chosen on some or all subdomains. In particular, if the DG method in [16] is employed on each subdomain, we obtain the same DG method on the whole $\Omega$. This latter case can also be obtained in the limit case where every subdomain consists of exactly one element. We also note that, in the hyperbolic case, only the upwind bilinear form $s_{b}(\cdot, \cdot)$ is non-vanishing on $\Gamma$.

Remark 3.2. We have chosen to impose Dirichlet conditions strongly; see the definition of the local spaces $V_{i}$. We can also impose them weakly, as is done for the SD method in [17] and the DG method in [16]. The results in the following sections remain valid in this case as well.

\section{A stability Result}

In this section, we prove a stability estimate for our approximate problem. We first recall an inverse inequality (see [24], Sect. 4.6.1 for a proof).

Lemma 4.1. Let the matrix $A$ be constant on each element. Then, the following inverse estimate holds, for every $u \in V$,

$$
\|\nabla \cdot(A \nabla u)\|_{0 ; t}^{2} \leq C_{\mathrm{inv}} \frac{p_{t}^{4}}{h_{t}^{2}}\|A \nabla u\|_{0 ; t}^{2}, \quad t \in \mathcal{T}_{i}, \quad i=1, \ldots, N,
$$

where $C_{\mathrm{inv}}$ only depends on the shape-regularity constants of the triangulations $\left\{\mathcal{T}_{i}\right\}$.

We define the following norm in $V$ :

$$
\|u\| \|^{2}:=\frac{1}{2} \sum_{i=1}^{N} \alpha_{i}+\frac{1}{2} \sum_{i=1}^{N} \beta_{i}+\rho,
$$

where

$$
\begin{aligned}
\alpha_{i} & :=\int_{\Omega_{i}}\left(A \nabla u \cdot \nabla u+\gamma u^{2}\right) \mathrm{d} x+\sum_{t \subset \Omega_{i}} \delta(t) \int_{t}(\mathbf{b} \cdot \nabla u)^{2} \mathrm{~d} x, \quad i=1, \ldots, N, \\
\beta_{i} & :=\int_{\partial_{-} \Omega_{i} \cap \Gamma}\left|\mathbf{b} \cdot \mathbf{n}_{i}\right|\lfloor u\rfloor^{2} \mathrm{~d} s, \quad i=1, \ldots, N, \\
\rho & :=\int_{\partial \Omega_{N} \cup \partial_{+} \Omega}|\mathbf{b} \cdot \mathbf{n}| u^{2} \mathrm{~d} s+\int_{\Gamma} \sigma[u]^{2} \mathrm{~d} s .
\end{aligned}
$$

We have the stability estimate.

Theorem 4.1. Let (6) and (7) hold, and let $\delta$ satisfy

$$
0 \leq \delta(t) \leq \frac{1}{2} \min \left\{\frac{h_{t}^{2}}{C_{\text {inv }} p_{t}^{4} \bar{a}_{t}}, \frac{\gamma_{0}}{\bar{c}_{t}^{2}}\right\}, \quad t \in \mathcal{T}_{i}, \quad i=1, \ldots, N,
$$

with $C_{\mathrm{inv}}$ the constant defined in Lemma 4.1. If the hypotheses of Lemma 4.1 are satisfied then,

$$
a^{h}(u, u) \geq\|\| u \|^{2}, \quad u \in V .
$$


Proof. We consider (17) with $v=u$ and estimate each term. We have

$$
\begin{aligned}
& s_{r}(u, u)=\rho, \\
& s_{a}(u, u)=0 .
\end{aligned}
$$

For $i=1, \ldots, N$, we consider the local contributions

$$
\begin{aligned}
a_{i}^{h}(u, u) & =\int_{\Omega_{i}}\left(A \nabla u \cdot \nabla u+c u^{2}\right) \mathrm{d} x+\int_{\Omega_{i}}(\mathbf{b} \cdot \nabla u) u \mathrm{~d} x+\sum_{t \in \mathcal{T}_{i}} \delta(t) \int_{t} \hat{L} u(\mathbf{b} \cdot \nabla u) \mathrm{d} x \\
& =\int_{\Omega_{i}}\left(A \nabla u \cdot \nabla u+\gamma u^{2}\right) \mathrm{d} x+\sum_{t \in \mathcal{T}_{i}} \delta(t) \int_{t} L u(\mathbf{b} \cdot \nabla u) \mathrm{d} x+\frac{1}{2} \int_{\partial \Omega_{i}} \mathbf{b} \cdot \mathbf{n}_{i} u_{i}^{2} \mathrm{~d} s .
\end{aligned}
$$

We consider the second term on the right-hand side of (24). Using the definition of $L$ and the arithmeticgeometric mean inequality, we can write, for every element $t \in \mathcal{T}_{i}$,

$$
\begin{aligned}
\delta(t) \int_{t} L u(\mathbf{b} \cdot \nabla u) \mathrm{d} x & =\delta(t)\|\mathbf{b} \cdot \nabla u\|_{0 ; t}^{2}+\delta(t) \int_{t}(c u(\mathbf{b} \cdot \nabla u)-\nabla \cdot(A \nabla u)(\mathbf{b} \cdot \nabla u)) \mathrm{d} x \\
& \geq \delta(t)\|\mathbf{b} \cdot \nabla u\|_{0 ; t}^{2}-\frac{1}{2} \delta(t)\left(\int_{t}\left(2 c^{2} u^{2}+\frac{1}{2}(\mathbf{b} \cdot \nabla u)^{2}+2|\nabla \cdot(A \nabla u)|^{2}+\frac{1}{2}(\mathbf{b} \cdot \nabla u)^{2}\right) \mathrm{d} x\right) \\
& \geq \frac{1}{2} \delta(t)\|\mathbf{b} \cdot \nabla u\|_{0 ; t}^{2}-\delta(t)\left(\int_{t} c^{2} u^{2} \mathrm{~d} x+\frac{C_{\text {inv }} p_{t}^{4}}{h_{t}^{2}} \int_{t}|A \nabla u|^{2} \mathrm{~d} x\right),
\end{aligned}
$$

where, for the last inequality, we have employed Lemma 4.1. Using (22) and summing over the elements in $\mathcal{T}_{i}$, we obtain

$$
\begin{aligned}
\int_{\Omega_{i}}\left(A \nabla u \cdot \nabla u+\gamma u^{2}\right) \mathrm{d} x+\sum_{t \in \mathcal{T}_{i}} \delta(t) \int_{t} \hat{L} u(\mathbf{b} \cdot \nabla u) \mathrm{d} x \geq & \\
& \frac{1}{2} \int_{\Omega_{i}}\left(A \nabla u \cdot \nabla u+\gamma u^{2}\right) \mathrm{d} x+\frac{1}{2} \sum_{t \in \mathcal{T}_{i}} \delta(t)\|\mathbf{b} \cdot \nabla u\|_{0 ; t}^{2} .
\end{aligned}
$$

Using (24) and (25), and summing over the substructures, we find

$$
\sum_{i=1}^{N} a_{i}^{h}(u, u) \geq \frac{1}{2} \sum_{i=1}^{N} \alpha_{i}+\sum_{i=1}^{N} \frac{1}{2} \int_{\partial \Omega_{i}} \mathbf{b} \cdot \mathbf{n}_{i} u_{i}^{2} \mathrm{~d} s .
$$

There remains to bound $s_{b}(u, u)$ and the second term on the right-hand side of $(26)$. We have

$$
s_{b}(u, u)+\sum_{i=1}^{N} \frac{1}{2} \int_{\partial \Omega_{i}} \mathbf{b} \cdot \mathbf{n}_{i} u_{i}^{2} \mathrm{~d} s=\sum_{i=1}^{N}\left(\int_{\partial-\Omega_{i} \cap \Gamma}\left|\mathbf{b} \cdot \mathbf{n}_{i}\right|\lfloor u\rfloor u_{i} \mathrm{~d} s+\frac{1}{2} \int_{\partial \Omega_{i}} \mathbf{b} \cdot \mathbf{n}_{i} u_{i}^{2} \mathrm{~d} s\right) .
$$


We can write the right-hand side of (27) in the following way:

$$
\begin{aligned}
\sum_{i=1}^{N}\left(\frac{1}{2} \int_{\partial_{-} \Omega_{i} \cap \Gamma} \mathbf{b} \cdot \mathbf{n}_{i}\left(u_{i}^{2}-2 u_{i}^{2}+2 u_{i} u_{-i}\right) \mathrm{d} s+\frac{1}{2} \int_{\partial_{+} \Omega_{i}} \mathbf{b} \cdot \mathbf{n}_{i} u_{i}^{2} \mathrm{~d} s+\frac{1}{2} \int_{\partial-\Omega_{i} \backslash \Gamma} \mathbf{b} \cdot \mathbf{n}_{i} u_{i}^{2} \mathrm{~d} s\right)= \\
\sum_{i=1}^{N}\left(\frac{1}{2} \int_{\partial_{-} \Omega_{i} \cap \Gamma} \mathbf{b} \cdot \mathbf{n}_{i}\left(-u_{i}^{2}+2 u_{i} u_{-i}-u_{-i}^{2}\right) \mathrm{d} s+\frac{1}{2} \int_{\partial \Omega_{i} \backslash \Gamma} \mathbf{b} \cdot \mathbf{n}_{i} u_{i}^{2} \mathrm{~d} s\right) \\
=\frac{1}{2} \int_{\partial_{+} \Omega \cup \partial \Omega_{N}}|\mathbf{b} \cdot \mathbf{n}| u^{2} \mathrm{~d} s+\sum_{i=1}^{N} \frac{1}{2} \int_{\partial_{-} \Omega_{i} \cap \Gamma}\left|\mathbf{b} \cdot \mathbf{n}_{i}\right|\lfloor u\rfloor^{2} \mathrm{~d} s,
\end{aligned}
$$

where we have used the fact that the outflow boundary $\partial_{+} \Omega_{i}$ can be written as the union of some of the inflow parts of the adjacent substructures and $u$ vanishes on $\partial_{-} \Omega \cup \partial \Omega_{D}$. The proof is completed by combining (23), (25), (27) and (28).

We have the following corollary.

Corollary 4.1. Problem (18) is well-posed.

\section{A PRIORI ERROR ESTimates}

The purpose of this section is to derive an a priori bound for the approximation error. Throughout, we define $u$ and $u_{\mathrm{DG}}$ as the solutions of problem (1) and (18), respectively.

We first need some preliminary definitions and lemmas. The following approximation property can be found in $[5,24]$.

Lemma 5.1. Let $u \in H^{k_{i}}\left(\Omega_{i}\right), k_{i} \geq 0, i=1, \ldots, N$. Then, there exists $\Pi_{i} u=\Pi_{h_{i} ; p_{i}} u \in V_{i}$ and $C$, only depending on the shape-regularity constants of the triangulations $\left\{\mathcal{I}_{i}\right\}, s$, and the $\left\{k_{i}\right\}$, such that, if $t \in \mathcal{T}_{i}$,

$$
\left\|u-\Pi_{i} u\right\|_{s ; t} \leq C \frac{h_{t}^{m_{i}-s}}{p_{t}^{k_{i}-s}}\|u\|_{k_{i} ; t}, \quad 0 \leq s \leq m_{i}
$$

where $m_{i}:=\min \left\{p_{i}+1, k_{i}\right\}$.

It is possible to define a global operator $\Pi u$ on $V$ by

$$
\Pi u_{\left.\right|_{\Omega_{i}}}:=\Pi_{i} u, \quad i=1, \ldots, N .
$$

We decompose the error into two components:

$$
u-u_{\mathrm{DG}}=\eta+\xi
$$

where

$$
\begin{aligned}
& \eta:=u-\Pi u, \\
& \xi:=\Pi u-u_{\mathrm{DG}} .
\end{aligned}
$$

We next need a trace estimate. It can be easily proved using that for an element of unit diameter and a scaling argument. 
Lemma 5.2. Let $t \in \mathcal{T}_{i}$, for a fixed index $i=1, \ldots, N$, and $u \in H^{1}(t)$. Then

$$
\|u\|_{0 ; \partial t}^{2} \leq C\left(\|u\|_{0 ; t}\|\nabla u\|_{0 ; t}+h_{t}^{-1}\|u\|_{0 ; t}^{2}\right)
$$

where $C$ only depends on the aspect ratio of $t$.

The following inverse estimate can be found in [24] (Sect. 4.6.1).

Lemma 5.3. Let $t \in \mathcal{T}_{i}$, for an index $i=1, \ldots, N$, and $u \in V$. Then

$$
\|u\|_{0 ; \partial t}^{2} \leq C \frac{p_{t}^{2}}{h_{t}}\|u\|_{0 ; t}^{2}
$$

where $C$ only depends on the aspect ratio of $t$.

We proceed by first finding a bound for $\xi$.

Lemma 5.4. Let $\eta=u-\Pi u$ and $\xi=\Pi u-u_{\mathrm{DG}}$. Then there exists a constant $C$, that only depends on the aspect ratios of the elements of $\left\{\mathcal{T}_{i}\right\}$, such that

$$
\begin{aligned}
\||\xi|\|^{2} \leq & C \int_{\Gamma} \sigma[\eta]^{2} \mathrm{~d} s+C \int_{\partial_{+} \Omega \cup \partial \Omega_{N}}|\mathbf{b} \cdot \mathbf{n}| \eta^{2} \mathrm{~d} s \\
& +C \sum_{i=1}^{N}\left(\int_{\Omega_{i}}\left(A \nabla \eta \cdot \nabla \eta+\left(d+\delta^{-1}\right) \eta^{2}\right) \mathrm{d} x+\int_{\partial_{+} \Omega_{i} \cap \Gamma}\left|\mathbf{b} \cdot \mathbf{n}_{i}\right| \eta_{i}^{2} \mathrm{~d} s\right. \\
& \left.+\sum_{\substack{t \in \mathcal{T}_{i} \\
\bar{b}_{t} \neq 0}} \int_{t} \delta(t)(\hat{L} \eta)^{2} \mathrm{~d} x+\sum_{\substack{t \in \mathcal{T}_{i} \\
\partial t \cap \mp \neq \emptyset}} \int_{\partial t \cap \Gamma}\left(\frac{\bar{a}_{t} h_{t}}{p_{t}}\left|\nabla \eta_{i}\right|^{2}+\frac{\bar{a}_{t} p_{t}^{2}}{h_{t}}[\eta]^{2}\right) \mathrm{d} s\right),
\end{aligned}
$$

where

$$
d:=\frac{(c-\nabla \cdot \mathbf{b})^{2}}{\gamma}
$$

Proof. Using Theorem 4.1 and the fact that both $u$ and $u_{\mathrm{DG}}$ satisfy equation (18), we can write

$$
\||\xi|\|^{2} \leq a^{h}(\xi, \xi)=a^{h}\left(-\eta+u-u_{\mathrm{DG}}, \xi\right)=-a^{h}(\eta, \xi)
$$

It is then enough to find a bound for the right-hand side of (32):

$$
a^{h}(\eta, \xi)=\sum_{i=1}^{N} a_{i}^{h}(\eta, \xi)+s_{a}(\eta, \xi)+s_{b}(\eta, \xi)+s_{r}(\eta, \xi)
$$

We begin with the first term. For every substructure $\Omega_{i}$, we integrate by parts and obtain

$$
\begin{aligned}
& \int_{\Omega_{i}}(A \nabla \eta \cdot \nabla \xi+\mathbf{b} \cdot \nabla \eta \xi+c \eta \xi) \mathrm{d} x+\sum_{t \subset \Omega_{i}} \delta(t) \int_{t} \hat{L} \eta(\mathbf{b} \cdot \nabla \xi) \mathrm{d} x= \\
& \qquad \int_{\Omega_{i}}(A \nabla \eta \cdot \nabla \xi-\mathbf{b} \cdot \nabla \xi \eta+(c-\nabla \cdot \mathbf{b}) \eta \xi) \mathrm{d} x+\sum_{t \subset \Omega_{i}} \delta(t) \int_{t} \hat{L} \eta(\mathbf{b} \cdot \nabla \xi) \mathrm{d} x+\int_{\partial \Omega_{i}} \mathbf{b} \cdot \mathbf{n}_{i} \eta_{i} \xi_{i} \mathrm{~d} s .
\end{aligned}
$$


We can easily bound the contributions from the first four terms on the right-hand side of (34), using the Schwarz inequality:

$$
\begin{gathered}
\left(\sum_{i=1}^{N} \int_{\Omega_{i}} A \nabla \eta \cdot \nabla \xi \mathrm{d} x\right)^{2} \leq\|\mid \xi\|^{2} \sum_{i=1}^{N} \int_{\Omega_{i}} A \nabla \eta \cdot \nabla \eta \mathrm{d} x, \\
\left(\sum_{i=1}^{N} \int_{\Omega_{i}} \mathbf{b} \cdot \nabla \xi \eta \mathrm{d} x\right)^{2} \leq\|\mid \xi\|^{2} \sum_{i=1}^{N} \sum_{\substack{t \in \mathcal{T}_{i} \\
\bar{b}_{t} \neq 0}} \delta(t)^{-1} \int_{t} \eta^{2} \mathrm{~d} x, \\
\left(\sum_{i=1}^{N} \int_{\Omega_{i}}(c-\nabla \cdot \mathbf{b}) \xi \eta \mathrm{d} x\right)^{2} \leq\|\mid \xi\|^{2} \sum_{i=1}^{N} \int_{\Omega_{i}} d \eta^{2} \mathrm{~d} x, \\
\left(\sum_{i=1}^{N} \sum_{t \in \mathcal{T}_{i}} \delta(t) \int_{t} \hat{L} \eta(\mathbf{b} \cdot \nabla \xi) \mathrm{d} x\right)^{2} \leq\|\xi\|^{2} \sum_{i=1}^{N} \sum_{\substack{t \subset \Omega_{i} \\
\bar{b}_{t} \neq 0}} \delta(t) \int_{t}(\hat{L} \eta)^{2} \mathrm{~d} x .
\end{gathered}
$$

We next bound the last term on the right-hand side of $(34)$ and $s_{b}(\eta, \xi)$ in $(33)$. We have

$$
\begin{aligned}
& s_{b}(\eta, \xi)+\sum_{i=1}^{N} \int_{\partial \Omega_{i}} \mathbf{b} \cdot \mathbf{n}_{i} \eta_{i} \xi_{i} \mathrm{~d} s=\sum_{i=1}^{N}\left(-\int_{\partial_{-} \Omega_{i} \cap \Gamma} \mathbf{b} \cdot \mathbf{n}_{i}\lfloor\eta\rfloor \xi_{i} \mathrm{~d} s+\int_{\partial \Omega_{i}} \mathbf{b} \cdot \mathbf{n}_{i} \eta_{i} \xi_{i} \mathrm{~d} s\right) \\
& =\sum_{i=1}^{N}\left(\int_{\partial_{-} \Omega_{i} \cap \Gamma} \mathbf{b} \cdot \mathbf{n}_{i}\left(-\eta_{i} \xi_{i}+\eta_{-i} \xi_{i}+\eta_{i} \xi_{i}\right) \mathrm{d} s\right. \\
& \left.+\int_{\partial_{+} \Omega_{i}} \mathbf{b} \cdot \mathbf{n}_{i} \eta_{i} \xi_{i} \mathrm{~d} s+\int_{\partial_{-} \Omega_{i} \backslash \Gamma} \mathbf{b} \cdot \mathbf{n}_{i} \eta_{i} \xi_{i} \mathrm{~d} s\right) \\
& =\sum_{i=1}^{N}\left(\int_{\partial_{-} \Omega_{i} \cap \Gamma} \mathbf{b} \cdot \mathbf{n}_{i}\left(\eta_{-i} \xi_{i}-\eta_{-i} \xi_{-i}\right) \mathrm{d} s+\int_{\partial \Omega_{i} \backslash \Gamma} \mathbf{b} \cdot \mathbf{n}_{i} \eta_{i} \xi_{i} \mathrm{~d} s\right) \\
& =\sum_{i=1}^{N} \int_{\partial_{-} \Omega_{i} \cap \Gamma} \mathbf{b} \cdot \mathbf{n}_{i}\lfloor\xi\rfloor \eta_{-i} \mathrm{~d} s+\int_{\partial_{+} \Omega \cup \partial \Omega_{N}} \mathbf{b} \cdot \mathbf{n} \eta \xi \mathrm{d} s,
\end{aligned}
$$

where we have used the fact that the outflow boundary $\partial_{+} \Omega_{i}$ can be written as the union of some of the inflow parts of the adjacent substructures and $\eta$ and $\xi$ vanish on $\partial_{-} \Omega \cup \partial \Omega_{D}$. The two terms on the right-hand side of (36) can easily be bounded using the Schwarz inequality, and we obtain

$$
\left(s_{b}(\eta, \xi)+\sum_{i=1}^{N} \int_{\partial \Omega_{i}} \mathbf{b} \cdot \mathbf{n}_{i} \eta_{i} \xi_{i} \mathrm{~d} s\right)^{2} \leq \|\left.|\xi|\right|^{2}\left(\sum_{i=1}^{N} \int_{\partial_{+} \Omega_{i} \cap \Gamma}\left|\mathbf{b} \cdot \mathbf{n}_{i}\right| \eta_{i}^{2} \mathrm{~d} s+\int_{\partial_{+} \Omega \cup \partial \Omega_{N}}|\mathbf{b} \cdot \mathbf{n}| \eta^{2} \mathrm{~d} s\right)
$$


We now consider the third term in (33). We first note that

$$
\left|s_{a}(\eta, \xi)\right| \leq \sum_{i=1}^{N} \sum_{\substack{t \in \mathcal{T}_{i} \\ \partial t \cap \Gamma \neq \emptyset}} \int_{\partial t \cap \Gamma}\left(|[\xi]|\left|A \nabla \eta_{i} \cdot \mathbf{n}\right|+\left|A \nabla \xi_{i} \cdot \mathbf{n}\right||[\eta]|\right) \mathrm{d} s .
$$

After applying Schwarz inequality, we obtain

$$
\begin{aligned}
s_{a}(\eta, \xi)^{2} \leq & \left(\sum_{i=1}^{N} \sum_{\substack{t \in \mathcal{T}_{i} \\
\partial t \cap \Gamma \neq \emptyset}} \int_{\partial t \cap \Gamma} \sigma[\xi]^{2} \mathrm{~d} s\right)\left(\sum_{i=1}^{N} \sum_{\substack{t \in \mathcal{T}_{i} \\
\partial t \cap \Gamma \neq \emptyset}} \int_{\partial t \cap \Gamma} \frac{\bar{a}_{t}^{2}}{\sigma}\left|\nabla \eta_{i}\right|^{2} \mathrm{~d} s\right) \\
& +\left(\sum_{i=1}^{N} \sum_{\substack{t \in \mathcal{T}_{i} \\
\partial t \cap \Gamma \neq \emptyset}} \int_{\partial t \cap \Gamma} \frac{\bar{a}_{t}}{\epsilon_{t}}[\eta]^{2} \mathrm{~d} s\right)\left(\sum_{i=1}^{N} \sum_{\substack{t \in \mathcal{T}_{i} \\
\partial t \cap \Gamma \neq \emptyset}} \int_{\partial t \cap \Gamma} \epsilon_{t}\left(A \nabla \xi_{i}\right) \cdot \nabla \xi_{i} \mathrm{~d} s\right),
\end{aligned}
$$

where the $\left\{\epsilon_{t}\right\}$ are arbitrary positive numbers. We consider the last term on the right-hand side of (39). By applying Lemma 5.3, we find

$$
\epsilon_{t} \int_{\partial t \cap \Gamma}\left(A \nabla \xi_{i}\right) \cdot \nabla \xi_{i} \mathrm{~d} s \leq C \epsilon_{t} \frac{p_{t}^{2}}{h_{t}} \int_{t}\left(A \nabla \xi_{i}\right) \cdot \nabla \xi_{i} \mathrm{~d} x
$$

Choosing then $\epsilon_{t}=h_{t} / p_{t}^{2}$ in (39) and using the definition of $\sigma$, we deduce

$$
s_{a}(\eta, \xi)^{2} \leq C\left|\|\xi \mid\|^{2}\left(\sum_{i=1}^{N} \sum_{\substack{t \in \mathcal{T}_{i} \\ \partial t \cap \Gamma \neq \emptyset}} \int_{\partial t \cap \Gamma}\left(\frac{\bar{a}_{t} h_{t}}{p_{t}}\left|\nabla \eta_{i}\right|^{2}+\frac{\bar{a}_{t} p_{t}^{2}}{h_{t}}[\eta]^{2}\right) \mathrm{d} s\right) .\right.
$$

We finally consider the forth term in (33). We have

$$
s_{r}(\eta, \xi)^{2} \leq\|\mid \xi\|^{2} \int_{\Gamma} \sigma[\eta]^{2} \mathrm{~d} s .
$$

The lemma is then proven by combining (32), (33), (34), (35), (37), (41) and (42).

Before proving our error bound we need to introduce an additional notation: If $t \in \mathcal{T}_{i}$, we define

$$
\left\{\frac{\bar{a} p^{2}}{h}\right\}_{t}:= \begin{cases}\max \left\{\frac{\bar{a}_{t^{\prime}} p_{t^{\prime}}^{2}}{h_{t^{\prime}}}\right\}, & \text { if } m_{n-1}(\partial t \cap \Gamma)>0 \\ \frac{\bar{a}_{t} p_{t}^{2}}{h_{t}}, & \text { otherwise, }\end{cases}
$$

where the maximum is taken over $t^{\prime}=t$ and all the elements $t^{\prime}$ such that

$$
m_{n-1}\left(\partial t \cap \partial t^{\prime} \cap \Gamma\right)>0 .
$$

We are now ready to prove our error bound. 
Theorem 5.1. Let $u$ and $u_{\mathrm{DG}}$ be the solutions of problem (1) and (8), and (18), respectively. If the hypotheses of Theorem 4.1 hold, then there exists $C$, that only depends on the shape-regularity constants of the $\left\{\mathcal{T}_{i}\right\}$, such that, if $u \in H^{k_{i}}\left(\Omega_{i}\right), i=1, \ldots, N$, then

$$
\left\|u-u_{\mathrm{DG}}\right\|^{2} \leq C \sum_{i=1}^{N} \sum_{t \in \mathcal{T}_{i}}\left\{\frac{\bar{a} p^{2}}{h}\right\}_{t} \frac{h_{t}^{2 m_{i}-1}}{p_{i}^{2 k_{i}-1}}\|u\|_{k_{i} ; t}^{2}+C \sum_{i=1}^{N}\left(B \frac{h_{i}^{2 m_{i}-1}}{p_{i}^{2 k_{i}-1}}+C_{i} \frac{h_{i}^{2 m_{i}}}{p_{i}^{2 k_{i}}}\right)\|u\|_{k_{i} ; \Omega_{i}}^{2},
$$

where $m_{i}:=\min \left\{p_{i}+1, k_{i}\right\}$, and, for $i=1, \ldots, N$,

$$
\begin{aligned}
B_{i} & :=\bar{b}_{i}\left(1+\|\delta\|_{\infty ; \Omega_{i}} \bar{b}_{i} \frac{p_{i}}{h_{i}}\right), \\
C_{i} & :=\left\|\gamma+d+\delta^{-1}\right\|_{\infty ; \Omega_{i}} .
\end{aligned}
$$

Proof. Using the triangle inequality, we have

$$
||\left|u-u_{\mathrm{DG}}\right|\|\leq|||\eta|\|+|||\xi||| .
$$

We employ Lemma 5.4 for the term in $\xi$, and then group and estimate the various contributions coming from the right-hand side of (30) and $\|\eta \eta\| \mid$. Using Lemma 5.1, we easily obtain, for $i=1, \ldots, N$,

$$
\int_{\Omega_{i}}\left((A \nabla \eta) \cdot \nabla \eta+\left(\gamma+d+\delta^{-1}\right) \eta^{2}\right) \mathrm{d} x \leq C \sum_{t \in \mathcal{T}_{i}}\left(\frac{\bar{a}_{t} h_{t}^{2 m_{i}-2}}{p_{i}^{2 k_{i}-2}}\|u\|_{k_{i} ; t}^{2}\right)+C\left\|\gamma+d+\delta^{-1}\right\|_{\infty ; \Omega_{i}} \frac{h_{i}^{2 m_{i}}}{p_{i}^{2 k_{i}}}\|u\|_{k_{i} ; \Omega_{i}}^{2} .
$$

We next consider the term

$$
\begin{aligned}
\sum_{\substack{t \in \mathcal{T}_{i} \\
\bar{b}_{t} \neq 0}} \delta(t) \int_{t}(\hat{L} \eta)^{2} \mathrm{~d} x & \leq 3 \sum_{\substack{t \in \mathcal{T}_{i} \\
\bar{b}_{t} \neq 0}} \delta(t) \int_{t}\left((\nabla \cdot(A \nabla \eta))^{2}+(\mathbf{b} \cdot \nabla \eta)^{2}+c \eta^{2}\right) \mathrm{d} x \\
& \leq C \sum_{\substack{t \in \mathcal{T}_{i} \\
\bar{b}_{t} \neq 0}} \delta(t)\left(\left(C_{\text {inv }} \frac{p_{t}^{4}}{h_{t}^{2}}\right) \bar{a}_{t}^{2} \frac{h_{t}^{2 m_{i}-2}}{p_{t}^{2 k_{i}-2}}+\bar{b}_{t}^{2} \frac{h_{t}^{2 m_{i}-2}}{p_{t}^{2 k_{i}-2}}+\bar{c}_{t}^{2} \frac{h_{t}^{2 m_{i}}}{p_{t}^{2 k_{i}}}\right)\|u\|_{k_{i} ; t}^{2},
\end{aligned}
$$

where we have used Lemmas 4.1 and 5.1. We then consider the boundary term

$$
\begin{aligned}
\sum_{i=1}^{N} \int_{\partial_{-} \Omega_{i} \cap \Gamma}\left|\mathbf{b} \cdot \mathbf{n}_{i}\right|\lfloor\eta\rfloor^{2} \mathrm{~d} s & \leq 2 \sum_{i=1}^{N} \int_{\partial_{-} \Omega_{i} \cap \Gamma}\left|\mathbf{b} \cdot \mathbf{n}_{i}\right|\left(\eta_{i}^{2}+\eta_{-i}^{2}\right) \mathrm{d} s \\
& =2 \sum_{i=1}^{N} \sum_{\substack{t \in \mathcal{T}_{i} \\
\partial t \cap \Gamma \neq \emptyset}} \int_{\partial t \cap \Gamma}\left|\mathbf{b} \cdot \mathbf{n}_{i}\right| \eta_{i}^{2} \mathrm{~d} s
\end{aligned}
$$

where we have used the property that the field $\mathbf{b}$ is continuous across the boundaries of the substructures; see condition (4). We then bound each local contribution using the trace estimates in Lemma 5.2 and Lemma 5.1. We obtain, for $t \in \mathcal{T}_{i}$,

$$
\int_{\partial t \cap \Gamma}\left|\mathbf{b} \cdot \mathbf{n}_{i}\right| \eta_{i}^{2} \mathrm{~d} s \leq C \bar{b}_{t}\left(\|\eta\|_{0 ; t}\|\nabla \eta\|_{0 ; t}+h_{t}^{-1}\|\eta\|_{0 ; t}^{2}\right) \leq C \bar{b}_{t} \frac{h_{t}^{2 m_{i}-1}}{p_{t}^{2 k_{i}-1}}\|u\|_{k_{i} ;}^{2}
$$


and, from (46),

$$
\sum_{i=1}^{N} \int_{\partial_{-} \Omega_{i} \cap \Gamma}\left|\mathbf{b} \cdot \mathbf{n}_{i}\right|\lfloor\eta\rfloor^{2} \mathrm{~d} s \leq C \sum_{i=1}^{N} \bar{b}_{i} \frac{h_{i}^{2 m_{i}-1}}{p_{i}^{2 k_{i}-1}}\|u\|_{k_{i} ; \Omega_{i}}^{2}
$$

We note that the terms

$$
\int_{\partial \Omega_{N} \cup \partial_{+} \Omega}|\mathbf{b} \cdot \mathbf{n}| u^{2} \mathrm{~d} s, \quad \text { and } \sum_{i=1}^{N} \int_{\partial_{+} \Omega_{i} \cap \Gamma}\left|\mathbf{b} \cdot \mathbf{n}_{i}\right| \eta_{i}^{2} \mathrm{~d} s
$$

can be bounded in a similar way.

Using Lemma 5.2 and Lemma 5.1, we also find

$$
\sum_{i=1}^{N} \sum_{\substack{t \in \mathcal{T}_{i} \\ \partial t \cap \Gamma \neq \emptyset}} \int_{\partial t \cap \Gamma}\left(\frac{\bar{a}_{t} h_{t}}{p_{t}}\left|\nabla \eta_{i}\right|^{2}+\frac{\bar{a}_{t} p_{t}^{2}}{h_{t}}[\eta]^{2}\right) \mathrm{d} s \leq C \sum_{i=1}^{N} \sum_{\substack{t \in \mathcal{T}_{i} \\ \partial t \cap \Gamma \neq \emptyset}}\left(\bar{a}_{t} \frac{h_{t}^{2 m_{i}-2}}{p_{i}^{2 k_{i}-2}}+\left\{\frac{\bar{a} p^{2}}{h}\right\}_{t} \frac{h_{t}^{2 m_{i}-1}}{p_{i}^{2 k_{i}-1}}\right)\|u\|_{k_{i} ; t}^{2}
$$

and, using the same argument,

$$
\int_{\Gamma} \sigma[\eta]^{2} \mathrm{~d} s \leq C \sum_{i=1}^{N} \sum_{\substack{t \in \mathcal{T}_{i} \\ \partial t \cap \Gamma \neq \emptyset}}\left\{\frac{\bar{a} p}{h}\right\}_{t} \frac{h_{t}^{2 m_{i}-1}}{p_{i}^{2 k_{i}-1}}\|u\|_{k_{i} ; t}^{2}
$$

The final estimate is proven by combining (44), (45), (47), (48) and (49).

In the particular case where the local meshes are quasi-uniform, we obtain the simpler bound

$$
\left\|u-u_{\mathrm{DG}}\right\|\left\|^{2} \leq C \sum_{i=1}^{N}\left(\left\{\frac{\bar{a} p^{2}}{h}\right\}_{i} \frac{h_{i}^{2 m_{i}-1}}{p_{i}^{2 k_{i}-1}}+B_{i} \frac{h_{i}^{2 m_{i}-1}}{p_{i}^{2 k_{i}-1}}+C_{i} \frac{h_{i}^{2 m_{i}}}{p_{i}^{2 k_{i}}}\right)\right\| u \|_{k_{i} ; \Omega_{i}}^{2},
$$

where, for $i=1, \ldots, N$,

$$
\left\{\frac{\bar{a} p^{2}}{h}\right\}_{i}:=\max _{j \in \mathcal{I}_{i}}\left\{\frac{\bar{a}_{j} p_{j}^{2}}{h_{j}}\right\}
$$

Finally, if

$$
\frac{\left(\bar{a}_{t^{\prime}} p_{t^{\prime}}^{2}\right) / h_{t^{\prime}}}{\left(\bar{a}_{t} p_{t}^{2}\right) / h_{t}}<\zeta
$$

for every couple of elements $\left(t, t^{\prime}\right)$ such that

$$
m_{n-1}\left(\partial t \cap \partial t^{\prime} \cap \Gamma\right)>0
$$

we can write

$$
\left\|u-u_{\mathrm{DG}}\right\|\left\|^{2} \leq C \sum_{i=1}^{N}\left(\bar{a}_{i} \frac{h_{i}^{2 m_{i}-2}}{p_{i}^{2 k_{i}-3}}+B_{i} \frac{h_{i}^{2 m_{i}-1}}{p_{i}^{2 k_{i}-1}}+C_{i} \frac{h_{i}^{2 m_{i}}}{p_{i}^{2 k_{i}}}\right)\right\| u \|_{k_{i} ; \Omega_{i}}^{2},
$$

with a constant $C$ that depends on $\zeta$. 


\section{The Case of a general Diffusion matrix $A$}

In this section, we briefly discuss the modifications of our method for the case of a matrix $A$ that is not constant on each element. The first modification has already been proposed in [17], and is necessary since Lemma 4.1 does not hold for a general $A$ :

On an element $t \subset \mathcal{T}_{i}$, the operator $\hat{L}$ employed in the stabilized bilinear form (11) and right-hand side (12) is now defined as

$$
\hat{L} u:=-\nabla \cdot\left(P_{t}(A \nabla u)\right)+\mathbf{b} \cdot \nabla u+c u,
$$

where $P_{t}: L^{2}(t)^{n} \longrightarrow \mathbb{P}_{p_{i}}(t)^{n}$ is the $L^{2}$-orthogonal projection onto $\mathbb{P}_{p_{i}}(t)^{n}$ (see [17], Sect. 3 ).

We then need to modify the bilinear form $s_{a}(\cdot, \cdot)$. Indeed, inequality (40), derived from Lemma 5.3, is not valid in general. We define this bilinear form in terms of local contributions and change the definition of the bracket operator $\langle\cdot\rangle$ :

$$
s_{a}(u, v):=\frac{1}{2} \sum_{E_{i j} \in \mathcal{E}_{H}} \sum_{\substack{t \in \mathcal{T}_{i} \\ t^{\prime} \in \mathcal{T}_{j}}} \int_{\partial t \cap \partial t^{\prime}}([u]\langle A \nabla v \cdot \mathbf{n}\rangle-\langle A \nabla u \cdot \mathbf{n}\rangle[v]) \mathrm{d} s,
$$

where

$$
\langle A \nabla v \cdot \mathbf{n}\rangle_{\left.\right|_{\partial t \cap \partial t^{\prime}}}:=\frac{1}{2}\left(P_{t}\left(A_{i} \nabla v_{i}\right) \cdot \mathbf{n}+P_{t^{\prime}}\left(A_{j} \nabla v_{j}\right) \cdot \mathbf{n}\right) .
$$

It is easy to prove that, with these modifications, a similar error bound as that proven in the previous section still holds; see, in particular, the proof of [17] (Th. 9).

As already mentioned, these modifications do not appear to be necessary in the numerical tests considered in the next section. In addition, a modification of the bilinear form $s_{a}(\cdot, \cdot)$ does not appear to be needed either for the original DG method (see [16], Ex. 2).

\section{NUMERICAL RESULTS}

In this section, we present some numerical results for the case of piecewise linear FE spaces $(p=1)$ in two dimensions, and leave the case of higher-order elements and three dimensional problems for a future work. We are primarily interested in estimating the convergence rates for different types of problems, the performance of our algorithm for different kinds of partitions into subdomains, and its robustness with respect to the penalization parameter $\sigma_{0}$ (see (16)). We will consider the error in the $|\| \cdot|||-$ and $L^{2}$-norm. For second order problems, we employ the modified energy norm norm

$$
\|u\|_{\mathrm{DG}}:=\|\| u \| \mid, \quad \text { with } \sigma=0,
$$

which is independent of the penalization parameter $\sigma$.

For the stabilization function $\delta(t)$, we have followed [18]. For every element $t$, let the local Peclet number be

$$
P e_{t}:=\frac{h_{t} \bar{b}_{t}}{2 \bar{a}_{t}}, \quad \text { for } \bar{a}_{t}>0
$$

and

$$
\delta(t):= \begin{cases}\frac{\tau h_{t}}{2 \bar{b}_{t}}, & \text { if } \bar{a}_{t}=0 \text { or } P e_{t} \geq 1, \\ \frac{\tau h_{t}^{2}}{4 \bar{a}_{t}}, & \text { if } P e_{t}<1 .\end{cases}
$$




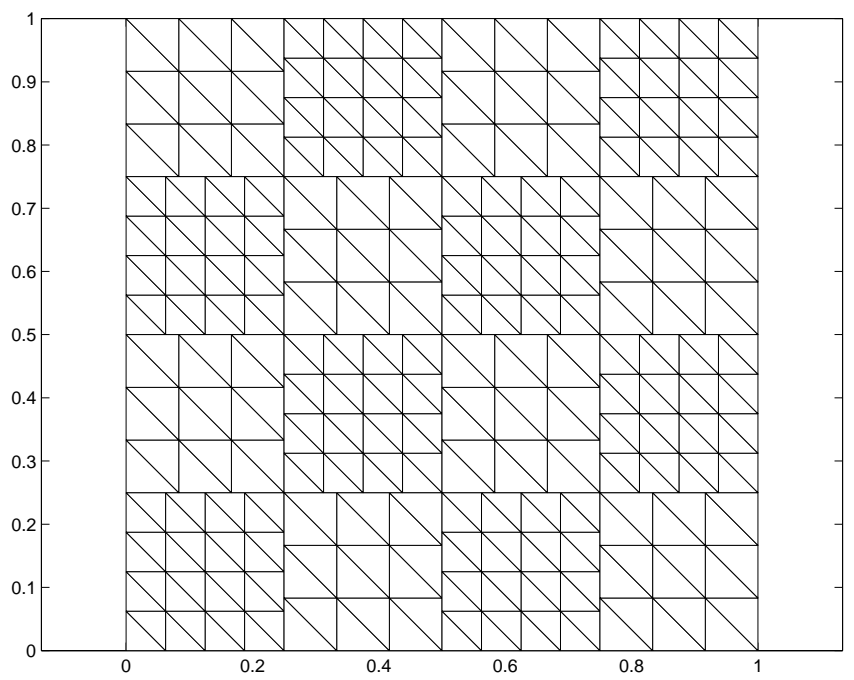

FIgURE 1. A conforming partition, with $m=4$, and a discretization with $n_{1}=4$ and $n_{2}=3$.

This definition can be generalized to the case $p>1$; see, e.g., [17]. In our experiments, we have considered the value $\tau=0.7$.

\subsection{Problems and partitions}

We consider problem $(1)$ and $\Omega=(0,1)^{2}$. We test two types of partitions:

Partition I: It is geometrically conforming and consists of $m \times m$ square substructures of equal size, with $m>1$. For a fixed $m$, we consider a checkerboard distribution for the local triangulations, consisting of two kinds of uniform triangular meshes. Let $n_{1}$ and $n_{2}$ be the corresponding numbers of steps and $h_{1}$ and $h_{2}$ their mesh-sizes. Figure 1 shows an example of this checkerboard-type discretization for $m=4$.

Partition II: It is geometrically non-conforming and consists of $m \times m$ equal square blocks. A block is made of five non-conforming subdomains and is shown in Figure 2, left, together with a possible triangulation. The four rectangular substructures in a block have the same mesh consisting of $2 \times n_{1} \times n_{2}$ triangles. The triangulation of the inner square consists of $2 \times n_{3} \times n_{3}$ elements. Figure 2, right, shows a partition for the case $m=2$ (four blocks and twenty subdomains). The number of subdomains is thus five times the number of blocks.

\subsection{Hyperbolic problem with analytic solution}

We consider problem (1), with

$$
\begin{aligned}
& A=0, \quad c=1+8 x y^{2}, \\
& \mathbf{b}=\left(2-(2 y-1)^{2}, 3-2 x\right),
\end{aligned}
$$

the exact solution

$$
u(x, y)=1+\sin \left(\pi x y^{2}\right)
$$

and the source term $f$ chosen consistently (see [16], Sect. 6.1).

Figure 3 shows the DG- and the $L^{2}$-norms of the error, versus the mesh-size $h$, for two geometrically conforming partitions (four and sixteen subdomains), on the left, and for two non-conforming ones (five and 

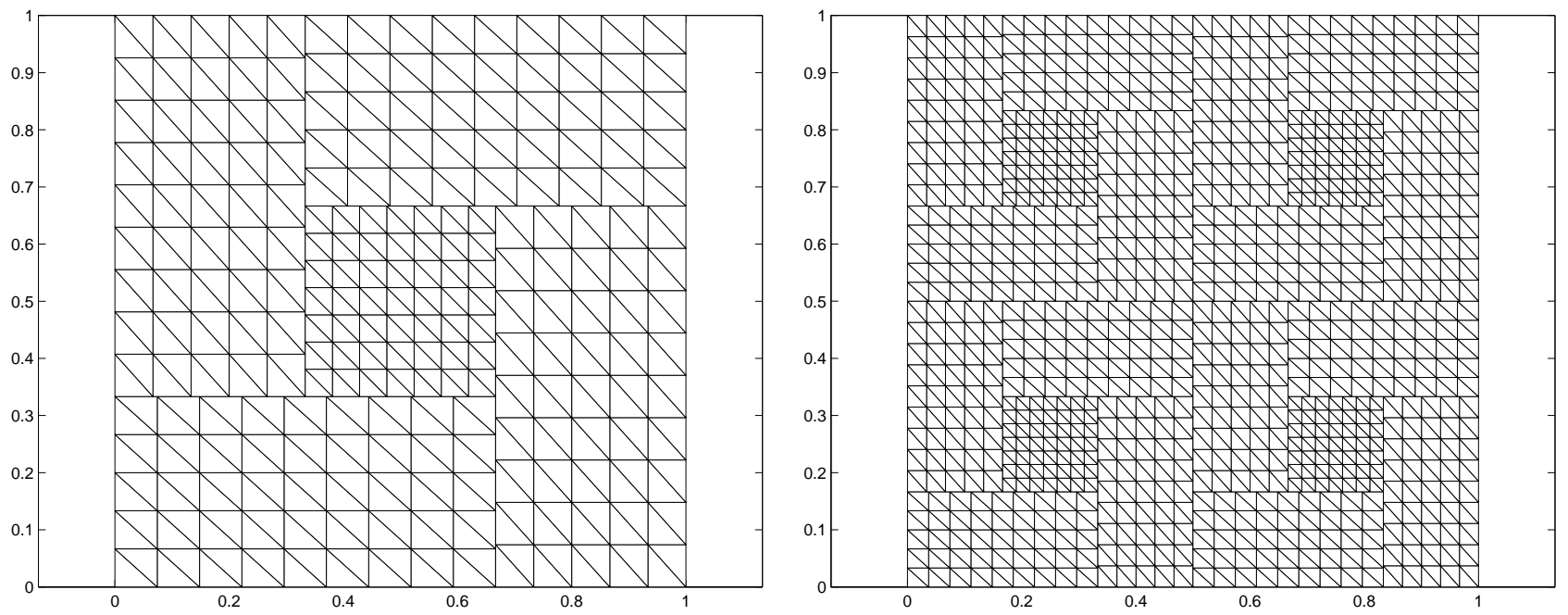

FIGURE 2. Left: a block consisting of five subdomains, employed for building a non-conforming partition $\left(n_{1}=9, n_{2}=5, n_{3}=7\right)$. Right: a non-conforming partition consisting of four blocks $\left(n_{1}=9, n_{2}=5, n_{3}=7\right)$.
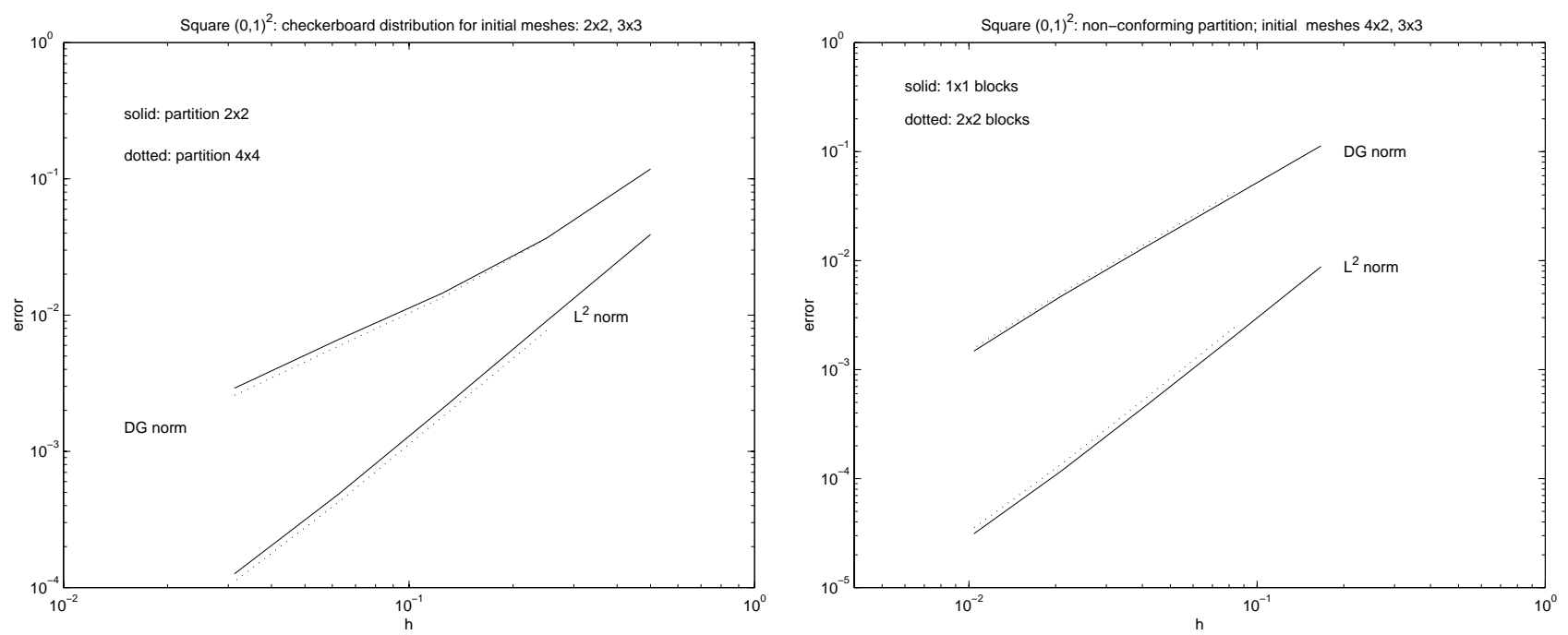

FIGURE 3. Hyperbolic problem: error in the DG- and $L^{2}$-norm versus the mesh-size. Conforming partitions with $m=2$ and $m=4$, and initial meshes with $n_{1}=2$ and $n_{2}=3$ (left), and non-conforming partitions with $m=1$ and $m=2$, and initial meshes with $n_{1}=4, n_{2}=2$, and $n_{3}=3$ (right).

twenty subdomains), on the right. Table 1 shows the slope calculated by least-square linear fitting of the curves in Figure 3. Our results suggest that the error $\left\|\left|u-u_{\mathrm{DG}} \|\right| \mid\right.$ converges to zero at the optimal rate $O\left(h^{3 / 2}\right)$ as $h$ tends to zero, in agreement with Theorem 5.1. In addition, our method also exhibits an optimal convergence rate $O\left(h^{2}\right)$ for $\left\|u-u_{\mathrm{DG}}\right\|_{0}$ for linear finite elements, as is the case of the original DG method in [16]. We note that the error appears to be independent of the partition into subdomains considered, and, in particular, that no deterioration of the solution is observed for geometrically non-conforming partitions. 
TABLE 1. Hyperbolic problem: calculated slope by least-square linear fitting of the plots in Figure 3.

\begin{tabular}{|l|cc|}
\hline & DG-norm & $L^{2}$-norm \\
\hline Part. I $(2 \times 2)$ & 1.57 & 2.08 \\
Part. I $(4 \times 4)$ & 1.59 & 2.04 \\
\hline Part. II $(1 \times 1)$ & 1.56 & 2.04 \\
Part. II $(2 \times 2)$ & 1.58 & 2.04 \\
\hline
\end{tabular}

TABLE 2. Hyperbolic problem with $x_{0}=\pi / 6$ : calculated slope by least-square linear fitting of the convergence plots in Figure 4.

\begin{tabular}{|l|cc|}
\hline & DG-norm & $L^{2}$-norm \\
\hline Part. I $(2 \times 2)$ & 1.13 & 1.36 \\
Part. I $(4 \times 4)$ & 1.13 & 1.46 \\
\hline Part. II $(1 \times 1)$ & 1.20 & 1.22 \\
Part. II $(2 \times 2)$ & 1.16 & 1.04 \\
\hline
\end{tabular}

\subsection{Hyperbolic problem with non-smooth solution}

We now consider the same hyperbolic problem as before, given by the coefficients (52), but with the exact solution given by

$$
u(x, y)= \begin{cases}\cos \left(\frac{\pi}{2}(2 y-1)\right), & x \leq x_{0} \\ \cos \left(\frac{\pi}{2}(2 y-1)\right)+\left(2\left(x-x_{0}\right)\right)^{\alpha} & , x>x_{0}\end{cases}
$$

with $x_{0} \in(0,1)$ (see [16], Sect. 6.1). It can be shown that $u$ belongs to $H^{\alpha+1 / 2}(\Omega)$, for $\alpha>0$, but does not belong to $H^{\alpha+1 / 2+\epsilon}(\Omega)$, for any $\epsilon>0$ (see [16]). We choose $\alpha=1$.

Our first set of results are for $x_{0}=\pi / 6 \sim 0.523$ and the same partitions as in the previous example. We note that the line $x=x_{0}$ cuts through the elements and the solution only belongs to $H^{3 / 2}\left(\Omega_{i}\right)$, for each subdomain $\Omega_{i}$. Figure 4 shows the DG- and the $L^{2}$-norms of the error versus the mesh-size $h$, and Table 2 the corresponding slopes calculated by least-square linear fitting. Our results are consistent with the predicted asymptotic behavior for the error $\left\|\left|u-u_{\mathrm{DG}} \|\right| \mid O(h)\right.$ as $h$ tends to zero and are in agreement with Theorem 5.1 . Concerning the error $\left\|u-u_{\mathrm{DG}}\right\|_{0}$, the curves in Figure 4 exhibit a more oscillating behavior and do not allow us to deduce an optimal rate of convergence $O\left(h^{3 / 2}\right)$. However, also in this case, the error $\left\|\left|u-u_{\mathrm{DG}} \|\right|\right.$ appears to be independent of the particular partition into subdomains considered.

Our second set of examples are for $x_{0}=1 / 2$. We consider Partition I with $m=2,4$ (four and sixteen subdomains), and Partition II with $m=2$ (twenty subdomains). In this case, the line $x=x_{0}$ does not cut through the subdomains and $u$ is analytic on each subdomain. Figure 5 shows the DG-and the $L^{2}$-norms of the error, versus the mesh-size $h$ and Table 3 the slope calculated by least-square linear fitting of the corresponding curves. Here, the optimal rate of convergence $O\left(h^{3 / 2}\right)$ for $\left\|\left|u-u_{\mathrm{DG}}\right|\right\|$ and $O\left(h^{2}\right)$ for $\left\|u-u_{\mathrm{DG}}\right\|_{0}$ is found, in agreement with Theorem 5.1. Also in this case, the errors appear to be independent of the particular partition considered. 

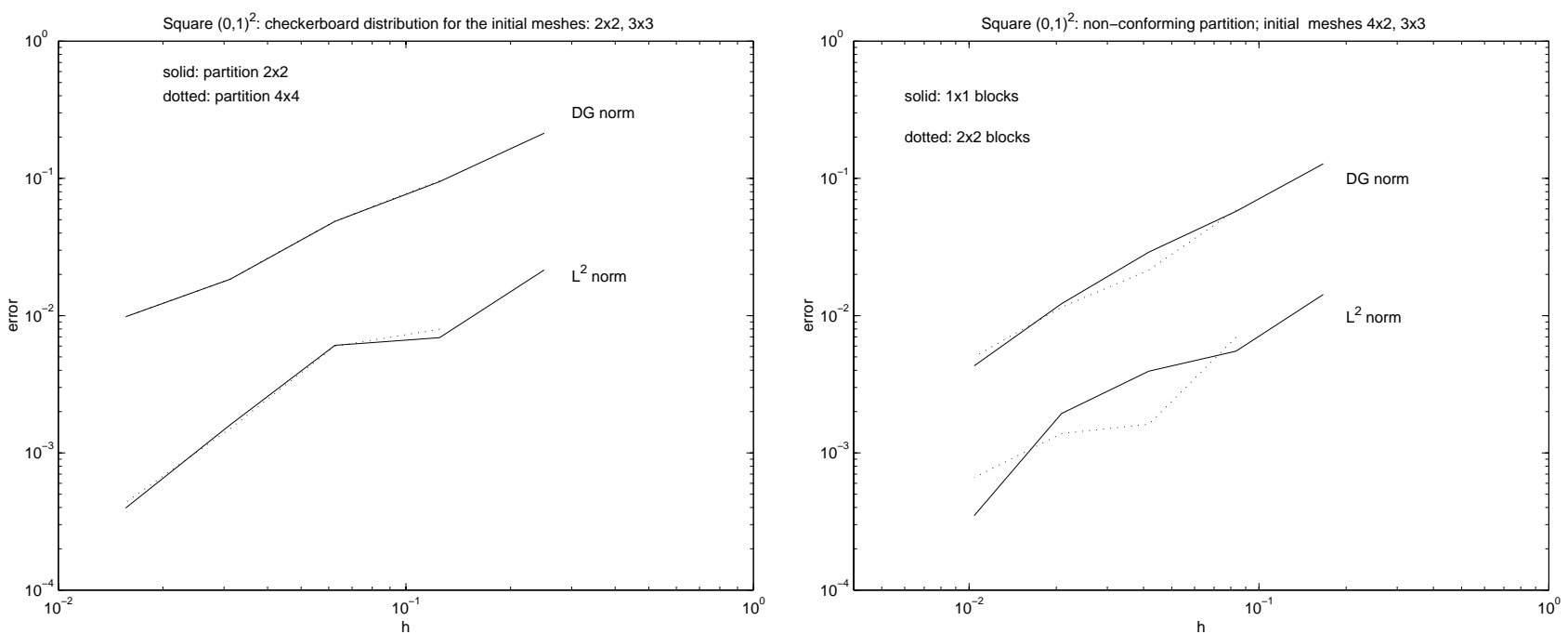

FiguRE 4. Hyperbolic problem with $x_{0}=\pi / 6$ : error in the DG- and $L^{2}$-norm versus the mesh-size. Conforming partitions with $m=2$ and $m=4$, and initial meshes with $n_{1}=2$ and $n_{2}=3$ (left), and non-conforming partitions with $m=1$ and $m=2$, and initial meshes with $n_{1}=4, n_{2}=2$, and $n_{3}=3$ (right).
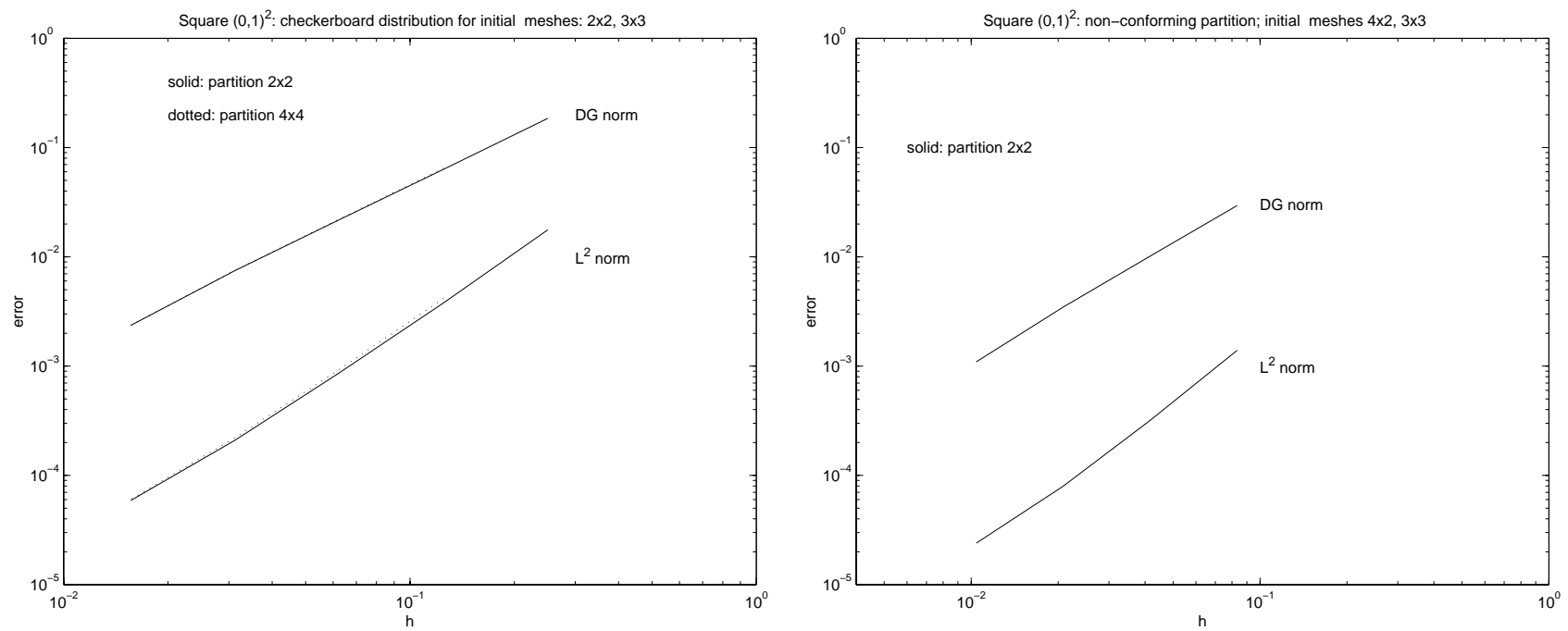

FiguRE 5. Hyperbolic problem with $x_{0}=1 / 2$ : error in the DG- and $L^{2}$-norm versus the mesh-size. Conforming partitions with $m=2$ and $m=4$, and initial meshes with $n_{1}=2$ and $n_{2}=3$ (left), and non-conforming partition with $m=2$, and initial mesh with $n_{1}=4, n_{2}=2$, and $n_{3}=3$ (right).

\subsection{Poisson problem}

We consider problem (1) with

$$
\begin{aligned}
& A=\left(\begin{array}{cc}
1+\sin (\pi x) & 0 \\
0 & 3-x
\end{array}\right), \\
& \mathbf{b}=0, \quad c=1+8 x y^{2},
\end{aligned}
$$


TABLE 3. Hyperbolic problem with $x_{0}=1 / 2$ : calculated slope by least-square linear fitting of the convergence plots in Figure 5.

\begin{tabular}{|l|cc|}
\hline & DG-norm & $L^{2}$-norm \\
\hline Part. I $(2 \times 2)$ & 1.57 & 2.06 \\
Part. I $(4 \times 4)$ & 1.59 & 2.04 \\
\hline Part. II $(2 \times 2)$ & 1.58 & 1.96 \\
\hline
\end{tabular}
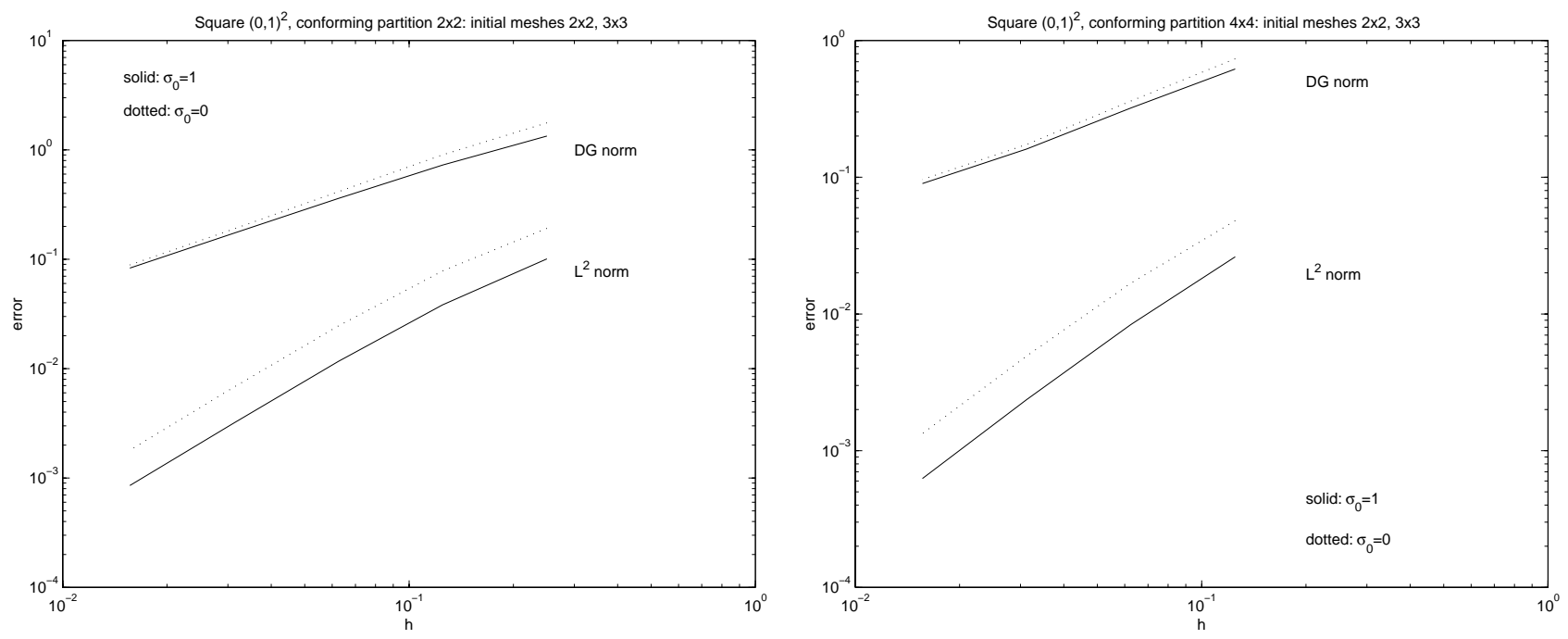

Figure 6. Elliptic problem: error in the DG- and $L^{2}$-norm versus the mesh-size. Conforming partitions with $m=2$ (left) and $m=4$ (right), and initial meshes with $n_{1}=2$ and $n_{2}=3$, for $\sigma_{0}=1$ and $\sigma_{0}=0$.

the exact solution

$$
u(x, y)=1+\sin \left(\pi x y^{2}\right),
$$

and the source term $f$ chosen consistently. We consider both cases with penalization $\left(\sigma_{0}=\sigma_{1}=1\right)$ and and without $\left(\sigma_{0}=\sigma_{2}=0\right)$; see (16).

Figure 6 shows the DG- and the $L^{2}$-norms of the error versus the mesh-size $h$, for two geometrically conforming partitions (four subdomains, on the left, sixteen, on the right). Figure 7 shows the convergence curves for two non-conforming partitions (five subdomains, on the left, twenty, on the right). In Table 4, we report the slope calculated by least-square linear fitting of the curves in Figures 6 and 7. Also in the elliptic case, our results suggest that the error $\left\|u-u_{\mathrm{DG}}\right\|_{\mathrm{DG}}$ converges to zero at an optimal rate $O(h)$ as $h$ tends to zero, in agreement with Theorem 5.1. Our results are also consistent with an optimal convergence rate $O\left(h^{2}\right)$ for $\left\|u-u_{\mathrm{DG}}\right\|_{0}$ for linear finite elements. These remarks are valid both for $\sigma_{0}=0$ and $\sigma_{0}=1$.

We also note that no appreciable degradation in the error $\left\|u-u_{\mathrm{DG}}\right\|_{\mathrm{DG}}$ is observed if no penalization term is employed $\left(\sigma_{0}=0\right)$. A slight degradation is observed in $\left\|u-u_{\mathrm{DG}}\right\|_{0}$. Our method appears to be robust with respect to the choice of the penalization parameter $\sigma_{0}$, at least for the test cases considered here. 

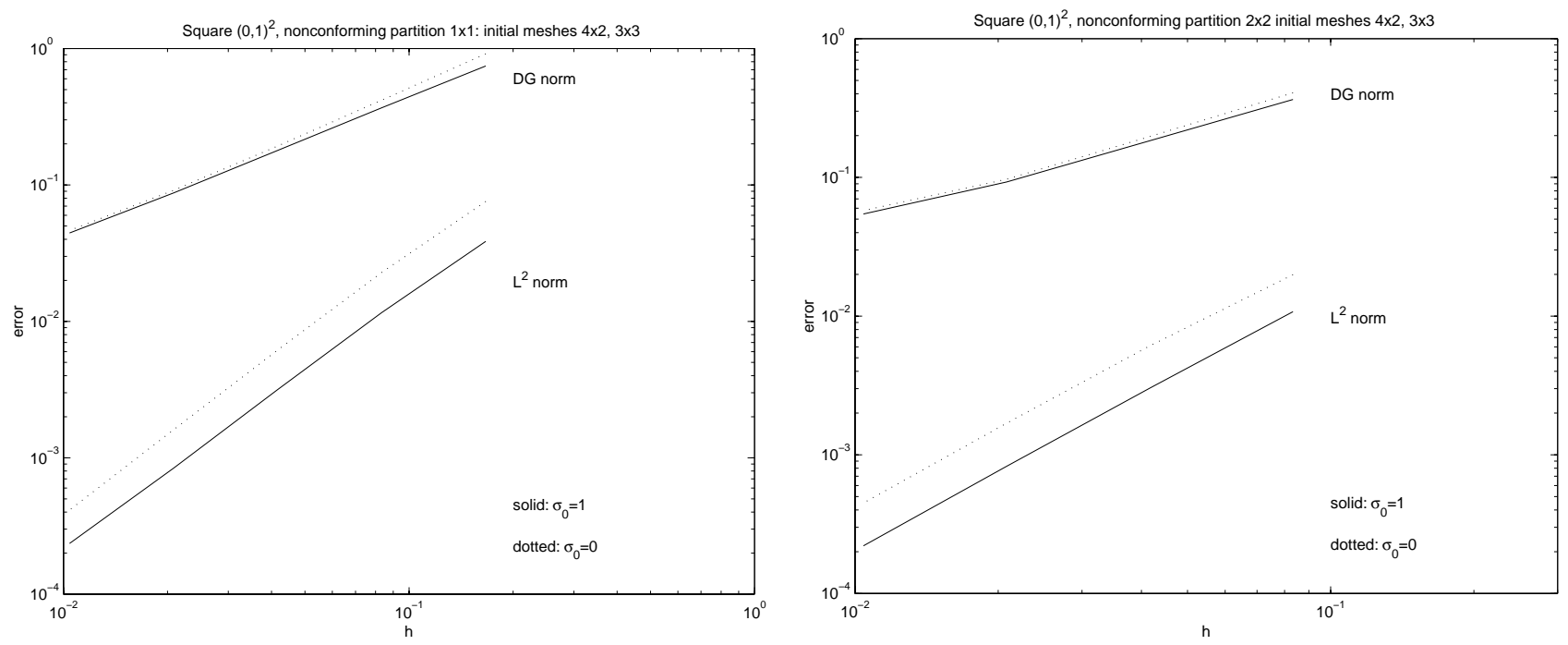

FiguRE 7. Elliptic problem: error in the DG- and $L^{2}$-norm versus the mesh-size. Nonconforming partitions with $m=1$ (left) and $m=2$ (right), and initial meshes with $n_{1}=4$, $n_{2}=3, n_{3}=3$, for $\sigma_{0}=1$ and $\sigma_{0}=0$.

TABLE 4. Elliptic problem: calculated slope by least-square linear fitting of the convergence plots in Figures 6 and 7.

\begin{tabular}{|l|cc|cc|}
\hline & DG-norm $\left(\sigma_{1}\right)$ & $L^{2}$-norm $\left(\sigma_{1}\right)$ & DG-norm $\left(\sigma_{2}\right)$ & $L^{2}$-norm $\left(\sigma_{2}\right)$ \\
\hline Part. I $(2 \times 2)$ & 1.01 & 1.74 & 1.09 & 1.70 \\
Part. I $(4 \times 4)$ & 0.93 & 1.78 & 0.99 & 1.73 \\
\hline Part. II $(1 \times 1)$ & 1.02 & 1.85 & 1.08 & 1.89 \\
Part. II $(2 \times 2)$ & 0.92 & 1.87 & 0.95 & 1.83 \\
\hline
\end{tabular}

\subsection{Coupled hyperbolic and singularly-perturbed elliptic problems}

We now consider a more complicated problem. We choose

$$
A= \begin{cases}\left(\begin{array}{ll}
\epsilon & 0 \\
0 & \epsilon
\end{array}\right), & r \leq 1 / 4 \\
0, & \text { otherwise }\end{cases}
$$

where $r^{2}=(x-1 / 2)^{2}+(y-1 / 2)^{2}$ and $\epsilon \geq 0, \mathbf{b}=\left(2 y^{2}-4 x+1,1+y\right), c=f=0$. The streamlines of $\mathbf{b}$ enter the domain from the three sides $x=0, x=1$, and $y=0$, and, along these lines, we prescribe the solution

$$
u(x, y)= \begin{cases}0, & x=0,1 / 2<y \leq 1 \\ 1, & x=0,0 \leq y \leq 1 / 2 \\ 1, & 0 \leq x \leq 3 / 4, y=0 \\ 0, & 3 / 4<x \leq 1, y=0 \\ \sin ^{2}(\pi y), & x=1,0 \leq y \leq 1\end{cases}
$$

We have chosen $\sigma_{0}=1$. 

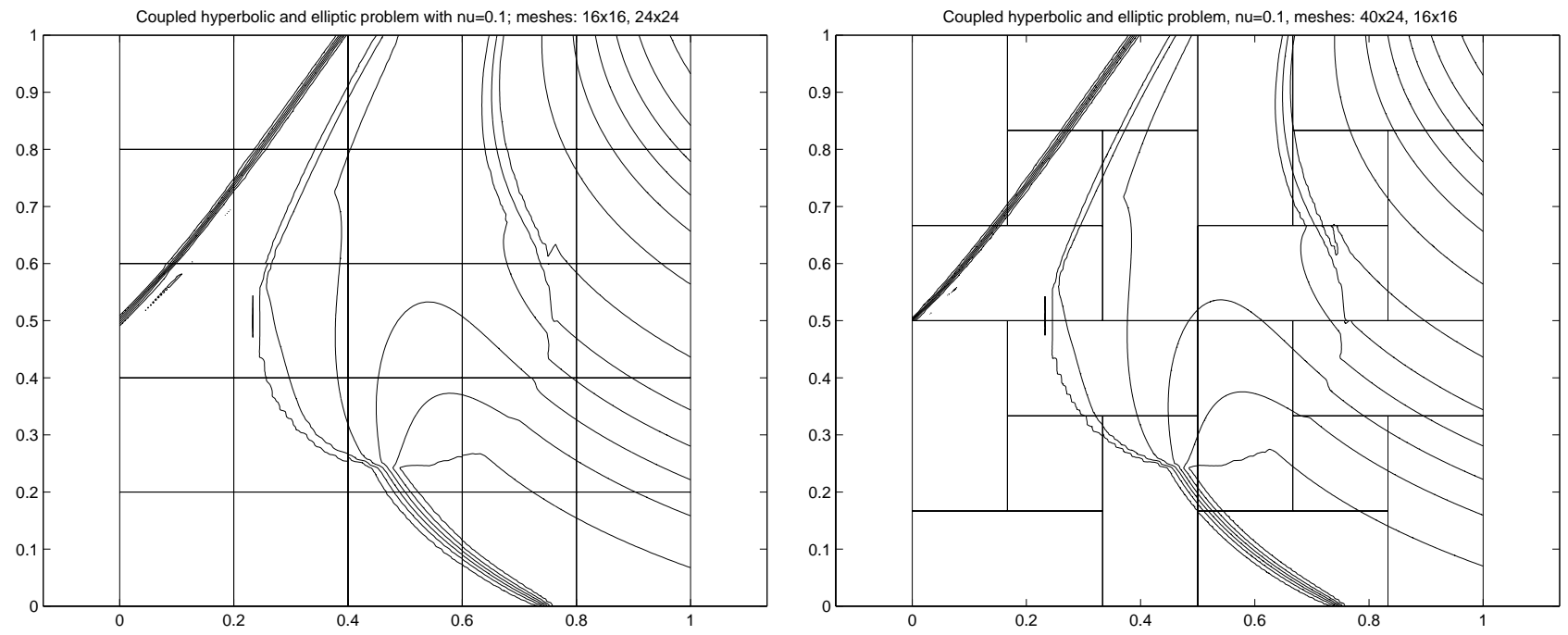

FIGURE 8. Coupled hyperbolic-elliptic problem with $\epsilon=0.1$ : conforming partition $(m=5,25$ subdomains) with $n_{1}=16, n_{2}=24,2 \times 10,240$ elements (left) and non-conforming partition ( $m=2$, 20 subdomains) with $n_{1}=40, n_{2}=24, n_{3}=16,2 \times 16,384$ elements (right).
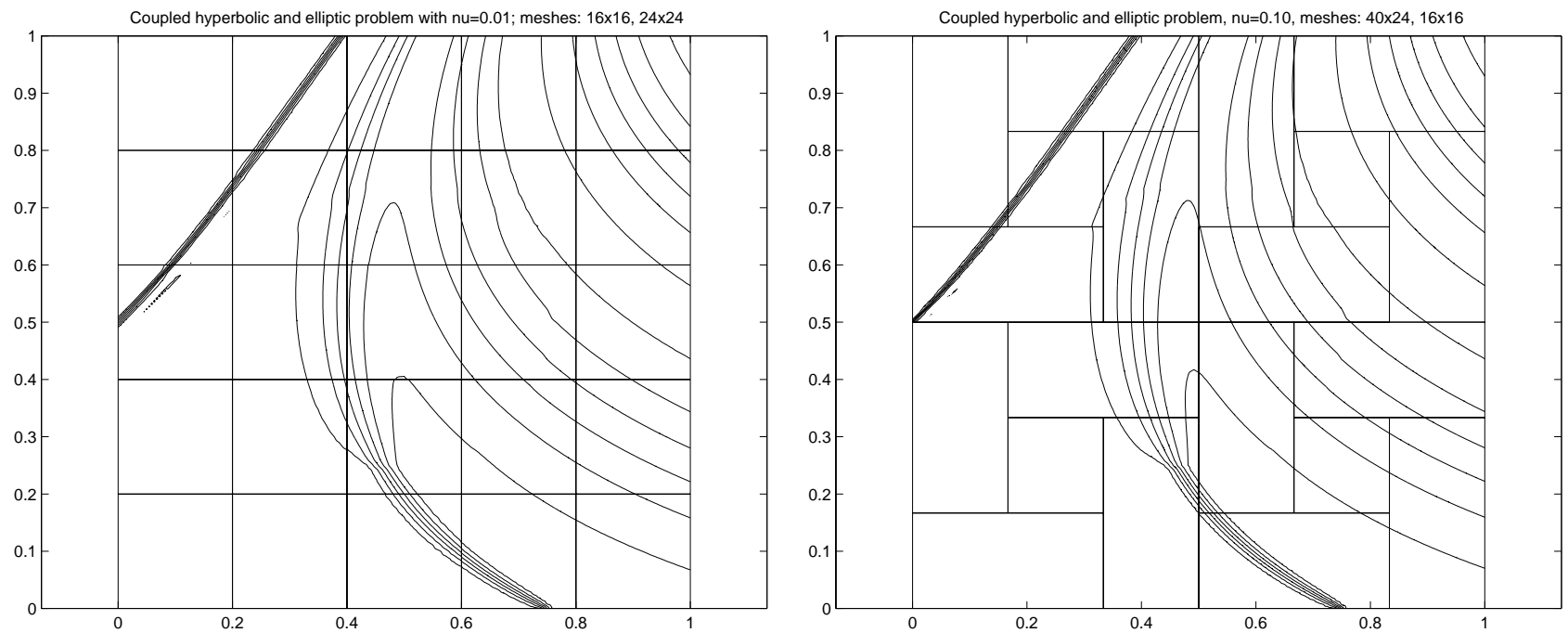

Figure 9. Coupled hyperbolic-elliptic problem with $\epsilon=0.01$ : conforming partition $(m=5$, 25 subdomains) with $n_{1}=16, n_{2}=24,2 \times 10,240$ elements (left) and non-conforming partition ( $m=2,20$ subdomains) with $n_{1}=40, n_{2}=24, n_{3}=16,2 \times 16,384$ elements (right).

This test problem is the same as that considered in [17] (Sect. 5.2). We note that, for $\epsilon>0$, the problem is elliptic in the circular region $r<1 / 4$ and hyperbolic in the remaining of $\Omega$. We consider a geometrically conforming partition (I), consisting of $5 \times 5$ subdomains and meshes given by $n_{1}=16, n_{2}=24(2 \times 10,240$ elements) and a non-conforming one, consisting of $2 \times 2$ blocks (20 subdomains) with meshes given by $n_{1}=40$, $n_{2}=24, n_{3}=16(2 \times 16,384$ elements $)$.

In Figures 8, 9, and 10, we show the contour plots of the solution $u$ for $\epsilon=0.1, \epsilon=0.01, \epsilon=0$, respectively, for the two partitions. As pointed out in [17], for $\epsilon=0.1$ and $\epsilon=0.01$, the boundary data is advected into the 

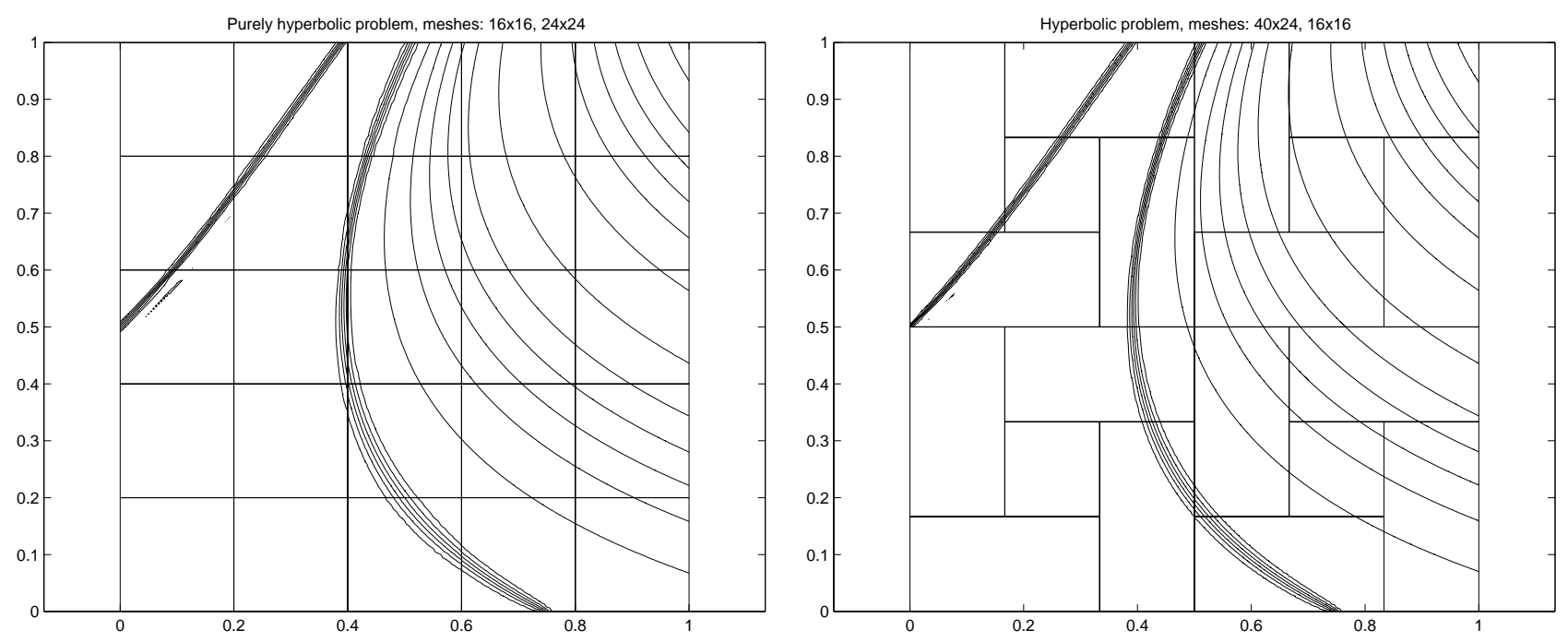

FIGURE 10. Hyperbolic problem $(\epsilon=0)$ : conforming partition $(m=5,25$ subdomains $)$ with $n_{1}=16, n_{2}=24,2 \times 10,240$ elements (left) and non-conforming partition $(m=2,20$ subdomains) with $n_{1}=40, n_{2}=24, n_{3}=16,2 \times 16,384$ elements (right).

hyperbolic region, then diffused in the elliptic region, and finally advected to the outflow boundary. We note that there are two discontinuity lines, due to the discontinuous boundary datum. For $\epsilon=0.01$, the diffusive effect is almost negligible. Our solutions appear to be in good agreement with those in [17], obtained with the SD method on a conforming triangulation, both for geometrically conforming and non-conforming partitions. In particular, we note that, for $\epsilon=0.1$ and $\epsilon=0.01$, the subdomain boundaries separate both hyperbolic and singularly-perturbed elliptic regions and that the contour lines are continuous across the subdomains.

\section{REFERENCES}

[1] Y. Achdou, The mortar element method for convection diffusion problems. C.R. Acad. Sci. Paris Sér. I Math. 321 (1995) $117-123$.

[2] Y. Achdou, C. Japhet, Y. Maday and F. Nataf, A new cement to glue non-conforming grids with Robin interface conditions: The finite volume case. Numer. Math. 92 (2002) 593-620.

[3] T. Arbogast, L.C. Cowsar, M.F. Wheeler and I. Yotov, Mixed finite element methods on non-matching multiblock grids. SIAM J. Numer. Anal. 37 (2000) 1295-1315.

[4] T. Arbogast and I. Yotov, A non-mortar mixed finite element method for elliptic problems on non-matching multiblock grids. Comput. Methods Appl. Mech. Engrg. 149 (1997) 255-265.

[5] I. Babuška and M. Suri, The hp version of the finite element method with quasi-uniform meshes. RAIRO Modél. Math. Anal. Numér. 21 (1987) 199-238.

[6] R. Becker and P. Hansbon, A finite element method for domain decomposition with non-matching grids. Technical Report $N^{\circ} 3613$, INRIA, January 1999.

[7] F. Ben Belgacem and Y. Maday, The mortar element method for three dimensional finite elements. RAIRO Modél. Math. Anal. Numér. 31 (1997) 289-302.

[8] F. Ben Belgacem and Y. Maday, Coupling spectral and finite element for second order elliptic three dimensional equations. SIAM J. Numer. Anal. 31 (1999) 1234-1263.

[9] A. Ben Abdallah, F. Ben Belgacem, Y. Maday and F. Rapetti, Mortaring the two-dimensional Nédélec finite element for the discretization of the Maxwell equations. $M^{2} A S$ (submitted).

[10] F. Ben Belgacem, The mortar element method with Lagrange multipliers. Numer. Math. 84 (1999) $173-197$.

[11] C. Bernardi, Y. Maday and A.T. Patera, A new non conforming approach to domain decomposition: The mortar element method, in Collège de France Seminar, H. Brezis and J.-L. Lions Eds., Pitman (1994).

[12] K.S. Bey, A. Patra and J.T. Oden, $h p$-version discontinuous Galerkin methods for hyperbolic conservation laws: A parallel adaptive strategy. Internat. J. Numer. Methods Engrg. 38 (1995) 3889-3908. 
[13] F. Brezzi and D. Marini, A three-field domain decomposition method, in Domain Decomposition Methods in Science and Engineering: The Sixth International Conference on Domain Decomposition, A. Quarteroni, Y.A. Kuznetsov, J. Périaux and O.B. Widlund Eds., AMS. Contemp. Math. 157 (1994) 27-34. Held in Como, Italy, June 15-19, 1992.

[14] F. Brezzi and D. Marini, Error estimates for the three-field formulation with bubble functions. Math. Comp. 70 (2001) $911-934$.

[15] B. Cockburn, G.E. Karniadakis and Chi-Wang Shu (Eds.), Discontinuous Galerkin Methods. Springer-Verlag, Lect. Notes Comput. Sci. Eng. 11 (2000).

[16] P. Houston, C. Schwab and E. Süli, Discontinuous $h p$-finite element methods for advection-diffusion-reaction problems. SIAM J. Numer. Anal. 39 (2002) 2133-2163.

[17] P. Houston and E. Süli, Stabilised $h p$-finite element approximation of partial differential equations with nonnegative characteristic form. Computing 66 (2001) 99-119. Archives for scientific computing. Numerical methods for transport-dominated and related problems, Magdeburg (1999).

[18] T.J.R. Hughes, L.P. Franca and G.M. Hulbert, A new finite element formulation for computational fluid dynamics: VIII. The Galerkin/least-squares method for advective-diffusive equations. Comput. Methods Appl. Mech. Engrg. 73 (1989) 173-189.

[19] C. Johnson, Numerical Solutions of Partial Differential Equations by the Finite Element Method. Cambridge University Press, Cambridge (1987).

[20] C. Johnson, U. Nävert and J. Pitkäranta, Finite element methods for linear hyperbolic problems. Comput. Methods Appl. Mech. Engrg. 45 (1984) 285-312.

[21] C. Johnson and J. Pitkäranta, An analysis of the discontinuous Galerkin method for a scalar hyperbolic equation. Math. Comp. 46 (1986) 1-26.

[22] P. Le Tallec and T. Sassi, Domain decomposition with nonmatching grids: Augmented Lagrangian approach. Math. Comp. 64 (1995) 1367-1396.

[23] A. Quarteroni and A. Valli, Numerical approximation of partial differential equations. Springer-Verlag, Berlin (1994).

[24] C. Schwab, $p$ - and hp-finite element methods. Oxford Science Publications (1998).

[25] R. Stenberg, Mortaring by a method of J.A. Nitsche, in Computational Mechanics: New trends and applications, S. Idelshon, E. Onate and E. Dvorkin Eds., Barcelona (1998). @CIMNE.

[26] M.F. Wheeler and I. Yotov, Physical and computational domain decompositions for modeling subsurface flows, in Tenth International Conference on Domain Decomposition Methods, J. Mandel, C. Farhat and X.-C. Cai Eds., AMS. Contemp. Math. 218 (1998) 217-228.

[27] B.I. Wohlmuth, A mortar finite element method using dual spaces for the Lagrange multiplier. SIAM J. Numer. Anal. 38 (2000) 989-1012.

[28] I. Yotov, Mixed Finite Element Methods for Flow in Porous Media. Ph.D. thesis, TICAM, University of Texas at Austin (1996).

[29] I. Yotov, A mixed finite element discretization on non-matching multiblock grids for a degenerate parabolic equation arising in porous media flow. East-West J. Numer. Math. 5 (1997) 211-230.

To access this journal online:

www.edpsciences.org 